Article

\title{
Method for Measuring the Information Content of Terrain from Digital Elevation Models
}

\author{
Lujin Hu ${ }^{1,2, *}$, Zongyi He ${ }^{1, \dagger}$, Jiping Liu ${ }^{2, \dagger}$ and Chunhua Zheng ${ }^{3, \dagger}$
}

1 School of Resource and Environmental Sciences, Wuhan University, Wuhan 430072, China; E-Mail: zongyihe@tom.com

2 Research Centre of Government Geographic Information System, Chinese Academy of Surveying and Mapping, Beijing 100830, China; E-Mail: liujp@casm.ac.cn

3 School of Computer Science and Technology, Xidian University, Xi' an 710071, China;

E-Mail: chzheng@mail.xidian.edu.cn

$\dagger$ These authors contributed equally to this work.

* Author to whom correspondence should be addressed; E-Mail: hulujin5388@163.com; Fax: +86-010-6388-0566.

Academic Editor: Raúl Alcaraz Martínez

Received: 29 July 2015 / Accepted: 12 October 2015 / Published: 16 October 2015

\begin{abstract}
As digital terrain models are indispensable for visualizing and modeling geographic processes, terrain information content is useful for terrain generalization and representation. For terrain generalization, if the terrain information is considered, the generalized terrain may be of higher fidelity. In other words, the richer the terrain information at the terrain surface, the smaller the degree of terrain simplification. Terrain information content is also important for evaluating the quality of the rendered terrain, e.g., the rendered web terrain tile service in Google Maps (Google Inc., Mountain View, CA, USA). However, a unified definition and measures for terrain information content have not been established. Therefore, in this paper, a definition and measures for terrain information content from Digital Elevation Model (DEM, i.e., a digital model or 3D representation of a terrain's surface) data are proposed and are based on the theory of map information content, remote sensing image information content and other geospatial information content. The information entropy was taken as the information measuring method for the terrain information content. Two experiments were carried out to verify the measurement methods of the terrain information content. One is the analysis of terrain information content in
\end{abstract}


different geomorphic types, and the results showed that the more complex the geomorphic type, the richer the terrain information content. The other is the analysis of terrain information content with different resolutions, and the results showed that the finer the resolution, the richer the terrain information. Both experiments verified the reliability of the measurements of the terrain information content proposed in this paper.

Keywords: terrain; information content; digital elevation models

\section{Introduction}

Terrain (or landform) represents various patterns of the land surface. Specifically, it reflects the relief of the Earth surface [1]. A digital elevation model (DEM) is the main approach to expressing terrain, and it is indispensable for topographic analysis and visualization [2-5]. Because terrain is an indispensable factor for modeling geographical processes (e.g., hydrologic processes [6], geologic processes [7], and natural hazard processes [8]), terrain information content can be applied to many fields along with other geo-spatial information. For example, we can evaluate the effectiveness of a rendered DEM for the render designer. Terrain information content can also be used for terrain generalization and visualization. We can use it to build level of detail (LOD) models for fast threedimensional terrain visualization in a virtual geographic environment. Moreover, it can be used for the choice of terrain multi-scale for geographical process modeling. Therefore, methods for measuring the terrain information content are significant and meaningful for topographic analysis and geographic process modeling. Terrain information content can be considered as one type of geospatial information content. The pioneering work for the geospatial information content can be traced to a map's information content [9-12], which was proposed by Sukhov. In his research, the map information is measured by the entropy of the map symbols. After his research, increasing numbers of researchers have paid attention to the information content of geospatial information [13-15] such as topographic information of maps [16], information content of contour maps [17], information content of remote sensing images [18-20], information content for thematic maps [21], information content for hydrological DEM [22], and information content for place names [23]. Geospatial information content of maps or images is important for the map designer to evaluate the effectiveness of the map, and it is also used for digital map generalization and data compression [11]. However, most of the geospatial information content is measured for the media of maps and remote sensing images, and there is not a unified definition or method for measuring terrain information from a DEM, which is still important for the geographic modeling, terrain generalization and evaluation of the quality of a rendered terrain service.

Because a unified definition and measurement methods have not been acquired for terrain information content, a definition and measurement method for terrain information content are proposed in this paper, and experiments are conducted to verify the measurement method. Specifically, this paper is dedicated to the following aspects:

(1) First, we propose the definition of the terrain information content, which is based on the theory of geospatial information content. The terrain information content is defined based on three 
aspects: the syntactic information content, the semantic information content, and the pragmatic information content.

(2) Second, we propose specific measurement methods for the terrain information content based on DEM. The methods for measuring the terrain information are expounded in detail from three aspects: the syntactic information content, the semantic information content, and the comprehensive information content.

(3) Finally, experiments are carried out to verify the measurement methods for the terrain information content. First, for geomorphic analysis of the terrain information content, we suppose that the more complex the terrain surface, the richer the terrain information. Second, for the resolution analysis, we suppose that the finer the resolution, the richer the terrain information.

\section{Study Basis}

Terrain information content is based on the geospatial information content, so the geospatial information content will be introduced first.

\subsection{Geospatial Information Content}

Most of the geospatial information content is based on the information theory, e.g., topographic information content [17] and spatial information content [15]. Thus, we will introduce the information content and the geospatial information content as follows.

\subsubsection{Introduction to Information Content}

Information content is a statistical description of the information characteristics. The definitions of information content are different in different fields. Generally speaking, in the field of information theory [24], information is defined as the uncertainty of the motion or exiting status of objects, which is measured by the information entropy, and information content is the uncertainty after obtaining the state of the things and is equal to the information entropy. In the communication field, information is focused on the transmission ability and anti-interference ability, and the information content is from the perspective of the observer (it is the information that transferred). In the field of control science, the concept of information includes the receiver response to the information, hence the information content is in the perspective of the receiver (it is the information that the receiver obtained and described). In the field of epistemology, information content is different according to the different receivers and their comprehensive abilities (it is the superior level of information content).

In epistemology, information can be divided into three levels from low to high [14]: syntactic information, semantic information and pragmatic information. Syntactic information is the information about the form and motion state of the objects, such as points, lines and polygons in a map. Semantic information is the implication of the specific motion state and other syntactic information. Pragmatic information is the value of the information for the information sink; it always depends on the demands of the information receivers, and it is difficult to evaluate [14,25]. 


\subsubsection{Geospatial Information Content}

Geospatial information contains all of the information that we can obtain from maps or other geographic media. Thus, with different geographic media, the geospatial information content can be grouped into various types. Among them, the most used geographic media are maps and remote sensing images. Thus, in this Section, we introduce map geospatial information content and remote sensing image information content:

(1) Map geospatial information content

If the geospatial information is defined as the spatial features and their diversity, which can be recognized from the map, the map geospatial information content can be defined as the level or the degree that can be measured for the spatial features and their diversity from the map. It represents the richness of the map information.

(2) Remote Sensing image information Content

With the advancement of remote sensing, the quality and the richness of the imagery at different spatial, spectral, and temporal resolutions is considered as the remote sensing image information content. The remote sensing image information content represents the usefulness and the richness of the remote sensor images.

\subsubsection{Basic Measurement Methods for Geospatial Information Content}

(1) Basic theories

In information theory, the concept of entropy is defined to determine the uncertainty of a message or an event. Entropy is the key measure for the information content. If there is an event $X$, and its value (event results) range is $X=\left\{x_{1}, \ldots, x_{n}\right\}$, the probability of every event result happens is $P=\left\{P\left(x_{1}\right), \ldots, P\left(x_{n}\right)\right\}$. The entropy for event $\mathrm{X}$ is (with units of bits):

$$
H(X)=-\sum_{i=1}^{n} P\left(x_{i}\right) * \log _{2}\left(P\left(x_{i}\right)\right)
$$

where $H(X)$ is the uncertainty of the event $X$, which is also the information content of the event, and $P\left(x_{i}\right)$ is the probability of the event with result $x_{i}$.

(2) Measurement method for map spatial information content.

Sukhov [9] first introduced information entropy as the main method for measuring map information content. He supposed that the total number of the symbols in the map is $\mathrm{N}$ and that there are $\mathrm{M}$ types of symbols in a map. Then, for every type of symbol, its probability can be calculated as:

$$
P_{i}=\frac{K_{i}}{N}
$$

where $P_{i}$ is the probability of the $i$-th type of symbol, and $K_{i}$ is the number of symbols of type $i$.

The map information content is defined as the sum of entropies of every type of symbol, which can be described as: 


$$
H(X)=-\sum_{i=1}^{M} P_{i}^{*} \log _{2}\left(\mathrm{P}_{i}\right)
$$

This is the first measurement method for the map information content. However, the distribution of the symbols in the map and spaces occupied by map symbols is not considered. The entropy of the symbols may be the same whether the symbols are highly assembled or widespread. Thus, Li [15] proposed that the Vonoroi region be used for every symbol to calculate the map spatial information content. In his research, three measures are proposed, which involve the metric information, the topological information and the thematic information. The metric information content is measured by the Vonoroi region areas, and the probability of the Vonoroi region is the ratio of this Vonoroi area to the total map area. It is shown as follows:

$$
P_{v o r . i}=\frac{S_{v o r . i}}{S}
$$

where Pvor.i is the probability of the Vonoroi region vor. $i, S_{\text {vor. } i}$ is the area of the Vonoroi region vor.i, and $S$ is the total area of the map.

The metric information content is the entropy of the Vonoroi region, expressed as:

$$
H(G M)=-\sum_{i=1}^{N} P_{v o r . i} * \log _{2}\left(P_{v o r . i}\right)
$$

where $H(G M)$ is the geometric information content, and $N$ is the total number of Vonoroi regions.

The topological information content is the average number for each Vonoroi region and is based on the idea of Neumann [17]. It can be calculated as:

$$
H(T)=\frac{N_{s}}{N}
$$

where $H(T)$ is the topological information content, $N_{s}$ is the sum of the neighbors for all of the map symbols, and $N$ is the total number of map symbols (as in formula 1).

The thematic information content is calculated by the probability of different Vonoroi types. The entropy of the Vonoroi types is defined as the thematic information content. It represents the differences of the symbols of the map. It can be calculated as:

$$
P_{\text {sym.i }}=\frac{N_{\text {type. } j}}{N_{\text {sym. } i}}
$$

where $P_{\text {sym.i }}$ is the probability of the symbol sym.i, $N_{\text {type.j }}$ is the number of the same symbol type type.j, and $N_{\text {sym.i }}$ is the total number of the neighbors of the symbol sym.i.

The thematic information content is the entropy of the Vonoroi neighbors, which can be calculated as:

$$
H(T M)=\sum_{\text {sym. } i=1}^{N} H(\text { sym. } i)=-\sum_{\text {sym. } i=1}^{N} \sum_{j=1}^{M} P_{\text {sym. } i} * \log _{2}\left(\mathrm{P}_{\text {sym. } . i}\right)
$$

where $H(T M)$ is the thematic information content, which is the sum of the Vonoroi neighbor entropy of all of the symbols. $H$ (sym.i) is the entropy of the symbol sym.i, $N$ is the total number of the symbols in the map, and $\mathrm{M}$ is the total types of symbols in the map.

(3) Measures for remote sensing image information content. 
The information content of the remote sensing image is mainly measured by the image pixel information entropy and some rectification for geometric distortion and the attribute equivocation [18,19]. Here, we simply introduce how to calculate the image pixel information content, which will be used for our study. The image pixel information entropy is calculated by the probability of the gray values of different bands in a color image, which can be shown as follows:

$$
H(P)=\sum_{i=1}^{N_{\text {band }}} H_{i}=-\sum_{i=1}^{N_{\text {band }}} \sum_{j=0}^{255} P_{j} * \log _{2}\left(P_{j}\right)
$$

where $H(P)$ is the pixel information entropy of the color image, $H_{i}$ is the entropy of the band $i$, and $P_{j}$ is the frequency of gray value $j$ in the total gray values, $N_{\text {band }}$ is the number of the band for a color image.

\subsection{Proposed Terrain Information Content}

A map can be considered as a medium carrying geographic information, and the map's information content was proposed for judging the map quality [15]. Hence, the rendered digital elevation model can also be regarded as the medium carrying the terrain information. In this way, when the terrain information is transformed in the internet or some service system, the terrain information can be calculated to justify the quality of the terrain information service (e.g., Map World of China, Google Maps) which provides the rendered terrain services for users. Then, the terrain information content can be defined similar to the map information content. The terrain information content is the information that can be obtained from DEM. Terrain information content can be described as three aspects in the epistemology, which are syntactic information, semantic information and pragmatic information.

(1) Terrain syntactic information content.

Syntactic information content is the information representing the motion state and the construction of the objects. It is the information that can be seen from the surface of the object, such as lines, points, and polygons from the thematic maps. Hence, the terrain syntactic information content can be defined as the information that can be directly seen from the rendered DEM surface. As shown in the rendered terrain in Figure 1, the following information can be directly seen from the surface of the rendered DEM: (a) geometric information such as the area and the resolution of the region; (b) color information, in which different colors represent different levels of elevation, as shown in Figure 1: the light brown and dark brown represent the high elevations, and the yellow and green colors represent the low elevations of the region. 


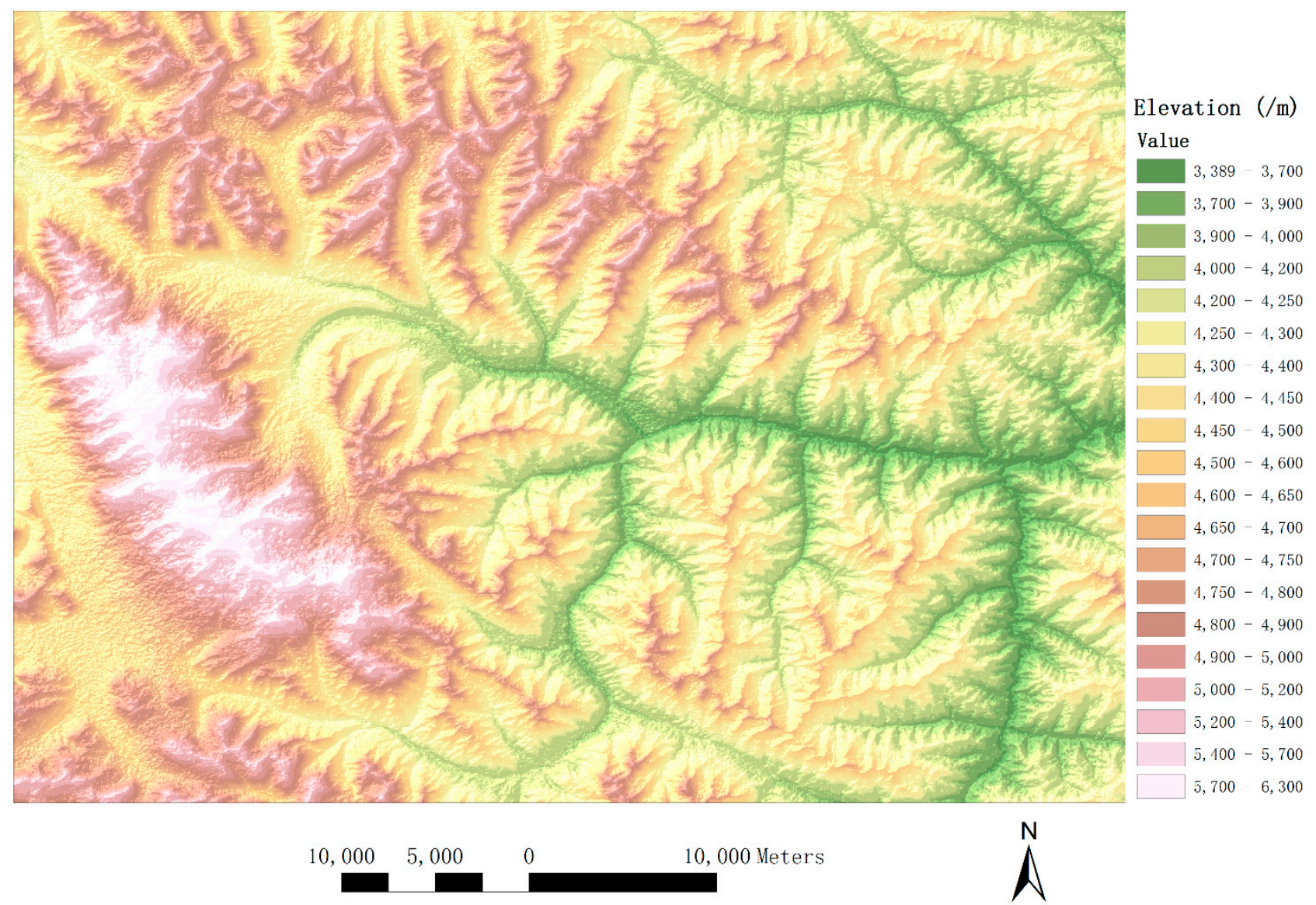

Figure 1. A rendered digital elevation model (DEM) of mountainous terrain with high altitude; the elevation values are rendered with different colors.

(2) Terrain semantic information content.

Semantic information is the information representing the meaning of the object's motion states, constructions and relations. It is the potential information that can be obtained from the syntactic information. Hence, the terrain semantic information can be defined as the potential information and the meaning of the terrain surface's formation. From a terrain surface, we can obtain a horizontal variation and a vertical variation, both of which can be viewed as the potential information that can be obtained indirectly from the DEM.

(3) Terrain pragmatic information content.

Pragmatic information content is the application of the information. The application of the information depends on the usefulness of the information to the thematic users. Therefore, the terrain pragmatic information content is the specific application and values for specific users. For example, for a hydrologist, the watershed unit information is generated from DEMs; for a soil researcher, the slope angle and slope length used in soil erosion models are two of the terrain factors. 


\section{Method for Measuring Terrain Information Content}

Specific measures are proposed for evaluating the terrain information content from a DEM, especially a rendered DEM. As depicted above, the definition of the terrain information content can be divided into three parts: syntactic information content, semantic information content and the pragmatic information content. Thus, the measures of the terrain information should be divided into these three parts, but because the pragmatic information depends on the uses and the application fields, we only propose pervasive measures in this paper for the terrain information content, and the pragmatic information content is not considered. Generally, in this paper, we propose measures for syntactic information content, semantic information content and the final comprehensive terrain information content.

\subsection{Terrain Syntactic Information Content}

Terrain syntactic information is the information that can be obtained from the terrain surface. Three types of information can be directly obtained from the rendered DEM: geometric information, terrain structure information, and rendered color information. Because every type of the information contributes equally to the syntactic information content, the terrain syntactic information content is the average value of the three values.

\subsubsection{Terrain Geometric Information Content}

Based on the theory of $\mathrm{Li}$ [15], geometric information should consider the space occupied by map symbols. In a raster DEM, every grid can be taken as a grid point with the grid center as its coordinate. Hence, in the terrain surface of DEM, the symbols are grid points (i.e., grid points in the DEM), the occupied space of each symbol is the same, and the measure for the geometric information content is defined as:

$$
H_{G E O}=\log _{2}(N)
$$

where $H_{G E O}$ is the geometric information content, and $N$ is the total numbers of grid points of the DEM.

\subsubsection{Terrain Structure Information Content}

The structure information represents the structural properties of a terrain (i.e., the properties of the elevation distribution). Because the terrain surface from a DEM is always a continuous surface, the elevation is distributed from low to high value in sequence. Hence, the structure of the terrain structure can be defined as a series of elevation subsets, which can be considered as the elevation unit with a specific method of elevation division (e.g., equal interval, natural breaks, geometric interval or user defined interval). In this paper, the unique integer elevation value is defined as the segmentation value for the elevation dataset. The partitioned elevation units are represented as a series of datasets, and $E_{i}$ is utilized to represent the $i$-th dataset, e.g., the integer elevation value of 10 is identified as the segmentation value for the datasets of $E_{9}$ and $E_{10}$, which are the datasets of $[9,10)$ and $[10,11)$. Hence, it doesn't matter whether the DEMs values are integer values or floating values when we partition the elevation to subsets for the entropy computation. The main differences between the source DEM data type are the probability computation of every elevation subset. e.g., for the elevation subset of $[10,11)$, 
the former is the frequency of the integer value of 10 , the latter it the total number of the value between this data range $[10,11)$.

The terrain structure information content can be defined as the entropy of probability of the elevation unit, i.e., the unique integer elevation value. The calculation formula is:

$$
H_{\text {STR }}=H\left(P_{1}, P_{2}, P_{3}, P_{4}, \ldots P_{n}\right)=-\sum_{i=1}^{N} P^{*} \log _{2}\left(P_{i}\right)=-\sum_{i=1}^{n} \frac{n_{i}}{N} \log _{2}\left(\frac{n_{i}}{N}\right)
$$

where $H_{S T R}$ is the terrain structure information content; $P_{1}, P_{2}, P_{3}, P_{4}, \ldots, P_{n}$ are the frequencies of the unique elevation unit $1,2,3,4, \ldots, \mathrm{n} ; n_{i}$ is the number of the elevation unit $i$ in the total terrain regions; and $N$ is the total number of elevation values (i.e., the elevation points).

\subsubsection{Rendered Color Information Content}

As the information content of the remote sensing images [19], the raster DEM also has the information content calculated from the rendered colors. In this paper, we defined this type of information content as the rendered color information, which is part of the terrain syntactic information. We describe the rendered information as two parts: one is the pixel entropy which is calculated from the red, green, and blue values of the rendered DEM, and the other is the pixel difference entropy, which describes the relationship of the center grid with its eight neighborhood pixels. Here, when the terrain information content is applied to different regions or different resolutions, all terrain regions should render with the same color ramp. The terrain rendered color information content is different from the terrain structure information content. It represents the color information from the rendered terrain maps and the color differences during the visual perception. When using the same color ramp, the rendered color information content represents the rendered information and the vision differences from different DEMs (i.e., different region of DEM). The rendered color information content can also be used for quality evaluation of different color rendering method, e.g., the terrain rendering tile map services in different electronic map navigation websites.

(1) Pixel information content.

This represents the properties of the rendered color maps. For the pixel entropy, we defined the value frequency to calculate the R, G, B entropy for the rendered color information, as in the following formula:

$$
H_{R G B}=[H(R)+H(G)+H(B)] / 3=\left[\sum_{i \text { band }=1}^{3} H\left(i_{\text {band }}\right)\right] / 3=\left[-\sum_{i \text { band }=1}^{3} \sum_{i=0}^{255} \frac{n_{i}}{255} \times \log _{2}\left(\frac{n_{i}}{255}\right)\right] / 3
$$

where $H_{R G B}$ is the pixel entropy for the rendered DEM, $H(R), H(G)$ and $H(B)$ represent the information entropy of the red, green and blue bands $i_{b a n d}$, is the band code of the rendered DEM, and the total number of bands is always three. $H\left(i_{\text {band }}\right)$ is the different band information entropy, and $n_{i}$ is the color pixel value, which is from 0 to 255 . 
(2) Pixel difference information content.

It represents the relationship of a pixel's value to those of its neighboring pixels, as shown in Figure 2. We calculated the frequency of the same pixel value among the eight neighbors as the probability of the current pixel value. The total pixel difference information is the average of all of the center pixels.

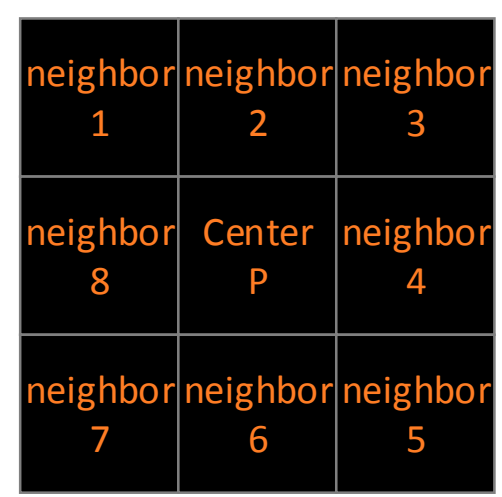

Figure 2. $3 \times 3$ window for the pixel difference information content calculation. The number in each window is the window's code.

For every center pixel $\mathrm{p}$, its RGB difference information content is defined as the entropy of its eight neighbor pixels. The total pixel difference information content is the average of the difference information content of all of the pixels, as in the following formula:

$$
\begin{aligned}
H_{\text {RGB_DIFF }} & =\left[H_{\text {DIFF }}(R)+H_{\text {DIFF }}(G)+H_{\text {DIFF }}(B)\right] / 3 \\
& =\sum_{\text {iband }}^{3} H_{\text {DIFF }}\left(i_{\text {band }}\right) \\
& =\left\{-\sum_{\text {iband }}^{3}\left[\sum_{i=0}^{N} \frac{n_{\text {nei_i }} i}{8} \times \log _{2}\left(\frac{n_{\text {nei_- }} i}{8}\right) / \mathrm{N}\right]\right\} / 3
\end{aligned}
$$

where $H_{R G B_{-} D I F F}$ is the pixel difference information content for the rendered DEM; $H_{D I F F}(R), H_{D I F F}(G)$, and $H_{D I F F}(B)$ are the information entropies of the red, green and blue bands; $n_{n e i} i$ is the number of the pixel value of pixel $i$ 's neighbor; and $N$ is the total number of elevation values.

There is a related problem that needs to be resolved before computing the pixel difference information content. For the margin pixels of the rendered DEM, not all of the eight neighbors exist. There is then the problem of how to compute the values of the neighbor's pixels. Thus, we need some special process. Two columns and two rows should be added to the original DEM, with the pixel values being the same as the nearest pixel values. As shown in Figure 3, the green region is the original DEM, and the value of every pixel is the red band pixel value. The yellow region is the added columns and rows, with the value of each pixel being the same as the nearest pixel's value in the original DEM. 


\begin{tabular}{|c|c|c|c|c|c|c|c|c|c|c|c|}
\hline 1 & 1 & 4 & 6 & 56 & 56 & 4 & 4 & 4 & 7 & 7 & 7 \\
\hline 1 & 1 & 4 & 6 & 56 & 56 & 4 & 4 & 4 & 7 & 7 & 7 \\
\hline 2 & 2 & 12 & 14 & 4 & 25 & 2 & 1 & 2 & 3 & 56 & 56 \\
\hline 3 & 3 & 14 & 13 & 23 & 7 & 4 & 5 & 43 & 52 & 53 & 53 \\
\hline 234 & 234 & 78 & 57 & 5 & 24 & 2 & 9 & 63 & 67 & 68 & 68 \\
\hline 35 & 35 & 67 & 56 & 6 & 21 & 6 & 6 & 7 & 87 & 90 & 90 \\
\hline 4 & 4 & 45 & 6 & 45 & 34 & 3 & 45 & 56 & 5 & 33 & 33 \\
\hline 3 & 3 & 7 & 56 & 35 & 6 & 5 & 78 & 3 & 232 & 234 & 234 \\
\hline 22 & 22 & 2 & 23 & 1 & 1 & 3 & 5 & 6 & 121 & 123 & 123 \\
\hline 34 & 34 & 1 & 35 & 7 & 7 & 6 & 9 & 8 & 3 & 45 & 45 \\
\hline 3 & 3 & 5 & 47 & 78 & 98 & 0 & 0 & 5 & 5 & 5 & 5 \\
\hline 3 & 3 & 5 & 47 & 78 & 98 & 0 & 0 & 5 & 5 & 5 & 5 \\
\hline
\end{tabular}

Figure 3. Margin value setting for processing the move window when we calculated the pixel difference information content with the gray value of one band.

(3) The rendered color information content.

This is the average value of the pixel information content and the pixel difference information content. It not only represents the pixel distribution properties but also the neighbor relation of the pixel distribution. The calculation formula is as follows:

$$
H_{R E N}=\left(H_{R G B}+H_{R G B}{ }_{D} \text { IFF }\right) / 2
$$

where $H_{R E N}$ is the rendered color information content.

\subsubsection{Terrain Syntactic Information Content}

The syntactic information content is the average value of the geometric information content, the structure information content, and the rendered color information content. Before the calculation, a unification of the value's magnitude is necessary. We should ensure that all types of the information value are set at the same magnitude. This can be shown as follows:

$$
H_{S Y N}=\left(H_{G E O}+H_{S T R}+H_{R E N}\right) / 3
$$

where $H_{S Y N}$ is the terrain syntactic information content.

\subsection{Terrain Semantic Information Content}

Terrain semantic information content is proposed to represent the potential meaning of terrain. Horizontal and vertical variations are the potential information of terrain. Slope angle of slope angle (SOS) and slope angle of slope aspect (SOA) [26] are chosen to represent the horizontal and vertical elevation changes of the terrain surface. If we use the other terrain information, such as relief degree, slope, roughness, most of them can only reflect some of the terrain information, but not the horizontal 
and the vertical totally. We should choose more than one terrain factor, but it is hard to find a suitable factor to just make up the parts that the current factor is not involved. But the SOA and SOS can reflect the horizontal variation and vertical variation comprehensively. Hence, the terrain semantic information content is computed as the entropy of the SOS value and the SOA value with a proper subset.

There are three main problems for computing the terrain semantic information content. One is how to compute the SOS and SOA. Another is how to partition the SOS and SOA values into subsets, and the third is how to compute the terrain semantic information content with the subsets of SOA and SOS.

\subsubsection{SOA and SOS}

To compute the values of SOS and SOA, we should first know the slope angle and slope aspect calculation models because they are the basis for the computation of SOA and SOS.

(1) Slope angle, as one of the most important parameters of terrain features, has been used in many aspects, such as hydrological models, soil erosion models, and land use planning. On the surface of the earth, the slope of a certain point is the elevation variability in the earth's surface $z=f(x, y)$ from the east-west ( $\mathrm{X}$ axis) to the north-south ( $\mathrm{Y}$ axis). Based on Grid DEM, slope is generally calculated at a local scale (such as a $3 \times 3$ moving window, as shown in Figure 4). The most used slope calculation model is the three-order inverse distance square weight difference, which has been used in ArcGIS software (Environmental Systems Research Institute Inc., Redlands, CA, USA). The variation rates of the surface at the horizontal $(d z / d x)$ and vertical $(d z / d y)$ directions from the center cell determine the slope. Slope is commonly measured in the units of degrees, and the data range is from 0 to 90 . The basic algorithm used to calculate the slope is [26-28]:

$$
\text { Slope }=\arctan \left(\sqrt{(d z / d x)^{2}+(d z / d y)^{2}}\right) * 180 / \pi
$$

If the letters from $a$ to $h$ represent the grid elevation in a $3 \times 3$ window. As shown in Figure $4, e$ is the center grid's elevation. Then the horizontal change $(d z / d x)$ and the vertical change $(d z / d y)$ can be computed as $[27,28]$ :

\begin{tabular}{|c|c|c|}
\hline$a$ & $b$ & $c$ \\
\hline$d$ & $e$ & $f$ \\
\hline$g$ & $h$ & $i$ \\
\hline
\end{tabular}

Figure 4. The $3 \times 3$ moving window for the slope computation.

$$
\begin{aligned}
& d z / d x=((c+2 f+i)-(a+2 d+g)) / 8 \times x_{-} \text {cellsize } \\
& d z / d y=((g+2 h+i)-(a+2 b+c)) / 8 \times y_{\text {_c cellsize }}
\end{aligned}
$$

(2) Slope aspect is the slope angle direction. With the grid DEM, all of the points can indicate the compass direction of the terrain surface. Methods of calculating slope aspect are based on the slope angle in accordance with the instructions of the compass; the output includes nine directions (as shown in 
Figure 5): median (Flat), north (N), northeast (NE), east (E), southeast (SE), south (S), northwest (NW), southwest (SW) and west (W). Every direction has an aspect value data range (with units of degrees), i.e., flat: Aspect $=-1$, north: Aspect $\in[0,22.5) \cup[337.5,360$ ), northeast: Aspect $\in[22.5,67.5)$, east: Aspect $\in[67.5,112.5)$, southeast: Aspect $\in[112.5,157.5)$, south: Aspect $\in[157.5,202.5)$, northwest: Aspect $\in[202.5,247.5)$, southwest: Aspect $\in[247.5,292.5)$, west: Aspect $\in[292.5,337.5)$. The output of the slope aspect is represented as these nine directions (mostly the result is a rendered aspect map, with nine colors to represent different directions). While the output of the slope aspect data is stored as a raster dataset, and every grid has a slope aspect value.

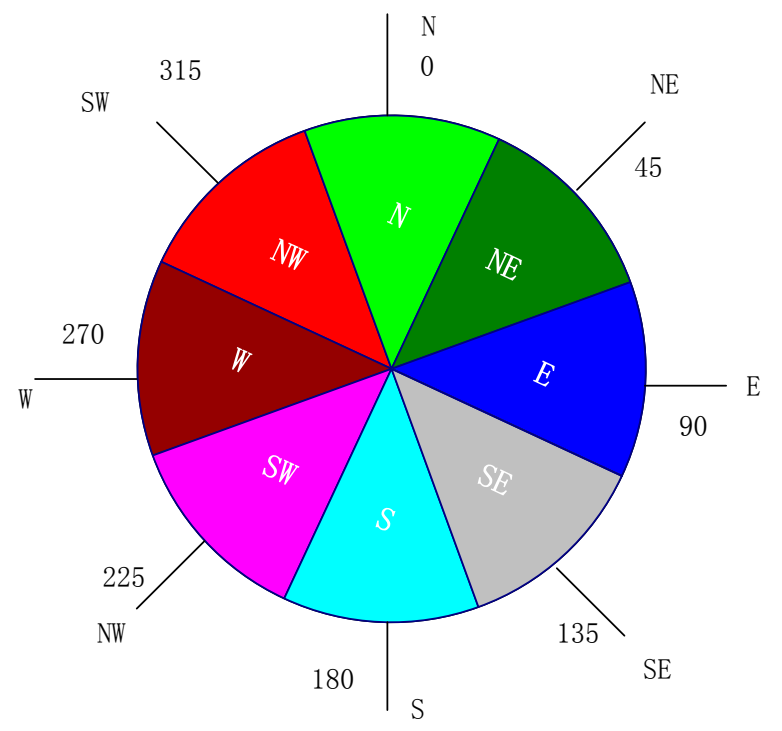

Figure 5. Slope aspect compass.

The slope aspect can be calculated as (units is degrees) [27,28]:

$$
\text { Aspect }=\arctan \left(\frac{d z / d y}{d z / d x}\right) * 180 / \pi
$$

The aspect value is then converted to compass direction values, according to the following rule $[27,28]$ :

If Aspect $<0$, then the new aspect value will be set as 90-Aspect; Else if Aspect $>90$, the new aspect value will be set as 360-Aspect +90 ; if $0<$ Aspect $<90$, the new aspect value will be set as 90-Aspect. Then according to the slope aspect direction compass and the slope aspect data range, the slope direction can be defined.

(3) SOS is the slope angle of the slope angle. It is based on the slope angle calculation model, after calculating the slope angle of the terrain surface, and then the calculated model is superimposed with the input of the slope angle. The SOS is the expression of the change of the ground elevation, which is characterized by the second order derivative of the surface elevation relative to the horizontal plane. Because of its simple calculation method, it is used to replace the curvature factor of a terrain profile. Because SOS is calculated by the slope degree calculation model, its output value range is the same as the slope degree, which is from 0 to 90 . The output value of SOS stores as a raster dataset, and every grid has a SOS value. 
If $a_{s}, b_{s}, c_{s}, d_{s}, e_{s}, f_{s}, g_{s}, h_{s}, i_{s}$ represent the slope degree of the $3 \times 3$ window (Figure 4), the SOS can be computed as [26]:

$$
S O S=\arctan \sqrt{\left[\frac{\left(c_{s}+2 f_{s}+i_{s}\right)-\left(a_{s}+2 d_{s}+g_{s}\right)}{8 \times x_{-} \text {cellsize }}\right]^{2}+\left[\frac{\left(g_{s}+2 h_{s}+i_{s}\right)-\left(a_{s}+2 b_{s}+c_{s}\right)}{8 \times y_{-} \text {cellsize }}\right]^{2}} * \frac{180}{\pi}
$$

(4) SOA refers to the slope aspect variability in the horizontal direction. The extraction method is based on the calculation of the surface slope angle. SOA can be obtained from the input of slope aspect in the slope angle calculation model.

If $a_{a}, b_{a}, c_{a}, d_{a}, e_{a}, f_{a}, g_{a}, h_{a}, i_{a}$ represent the slope aspect of the $3 \times 3$ window (Figure 4 ), the SOA can be computed as [26]:

$$
S O A=\arctan \sqrt{\left[\frac{\left(c_{a}+2 f_{a}+i_{a}\right)-\left(a_{a}+2 d_{a}+g_{a}\right)}{8 \times x_{-} \text {cellsize }}\right]^{2}+\left[\frac{\left(g_{a}+2 h_{a}+i_{a}\right)-\left(a_{a}+2 b_{a}+c_{a}\right)}{8 \times y_{-} \text {cellsize }}\right]^{2}} * \frac{180}{\pi}
$$

Because SOA is calculated with the slope degree model, its output data range is the same as the slope degree. Its value data range is from 0 to 90 . The output value of SOA is stored as a raster dataset, and every grid has a SOA value. There are some situations may met for the SOA computation, i.e., when two of the aspect value are 350 and 15 , according to the SOA calculation model, the aspect variation will be very large, but in fact, it is small, which can be seen from the slope aspect compass. So in reference [26], a rectification has been made for the SOA computation. The rectified method can be realized by two steps: First, the negative terrain should be calculated out, with the maximum elevation value minus every grid elevation value in the DEM raster dataset. Second, with the SOA calculation model (Equation (21)), the SOA values of the original DEM and the negative DEM can be computed out. We use $S O A_{\text {ori }}$ and $S O A_{\text {neg }}$ to represent them, respectively. Then the rectified SOA (SOA rec) can be computed as:

$$
S O A_{\text {rec }}=\frac{\left(S O A_{\text {ori }}+S O A_{\text {neg }}\right)-\left|S O A_{\text {ori }}-S O A_{\text {neg }}\right|}{2}
$$

The terrain information parameters SOA and SOS should be normalized. The extremum standardization method is used for the normalization. The normalization method calculation formula is as follows:

$$
\begin{gathered}
C\left(x_{i}\right)=\frac{x_{i}-x_{\min }}{x_{\max }-x_{\min }} \\
\left\{\begin{array}{l}
N_{S O S}=C(S O S) \\
N_{S O A}=C(S O A)
\end{array}\right.
\end{gathered}
$$

where $C\left(x_{i}\right)$ is the normalized of value $x_{i}, x_{\max }$ is the maximum value of $x_{i}, x_{\min }$ is the minimum value of $x_{i}, N_{S O S}$ is the normalized value of SOS, and $N_{S O A}$ is the normalized value of SOA. 


\subsubsection{Partition Method for SOS and SOA}

When we compute the terrain semantic information, the values of SOA and SOS should be partitioned into subsets to calculate the entropy. The normalized values of SOA and SOS are from 0 to 1 . Then, the normalized values of SOA and SOS are partitioned by a simple generated processing method. At the same time, the normalized value is enlarged 10 times and with an integer conversion. Then, the value's region is from 1 to 10 , and we only calculated the frequency of these integer values.

\subsubsection{Terrain Semantic Information Computation}

According to a certain subset partition method described above, we assumed that the SOS or SOA values are divided into $\mathrm{n}$ sub sets, where each subset occupies the area percentage, which is the same as the value frequency. The terrain semantic information content is the average of the entropy of the SOA and SOS values with their subsets:

$$
\begin{aligned}
& H(X)=-\sum_{i=0}^{n_{s e t}} \frac{N_{s u b_{-} i}}{N} \times \log \left(\frac{N_{s u b_{-} i}}{N}\right) \\
& H_{S E M}=[H(S O S)+H(S O A)] / 2
\end{aligned}
$$

where $H(X)$ is the entropy calculation function for the SOS and SOA values, $n_{\text {set }}$ is the number of the subset of the $\mathrm{X}$ values, $N$ is the total number of the elevation points, $N_{\text {sub }{ }_{-} i}$ is the number of the elevation points for the subset $s u b_{-} i$, and $H_{S E M}$ is the terrain semantic information content.

\subsection{General Information Content of Terrain}

The general information content of terrain is utilized to represent the comprehensive terrain information. With it, the transferred terrain map services can be qualified. Moreover, with the general information of terrain, different geomorphic terrain areas will be endowed with different terrain information content, i.e., the more complex the terrain, the larger the value of the general information content.

The general information content $\left(H_{T}\right)$ is set as the sum of terrain syntactic information and terrain semantic information content. Before the calculation, a unification of the value's magnitude is necessary. We should ensure that the two types of information values are set at the same magnitude. After that, we can use the following formula for the calculation:

$$
H_{T}=H_{S Y S}+H_{S E M}
$$

\subsection{Method Implementation}

Based on the method of measuring the terrain information content, a terrain information computation tool is realized with the $\mathrm{C}++$ programing language. There are nine key processes for extracting the terrain information content with the terrain information tools: (1) Data preparation: DEM data of the region, which we need to compute its terrain information content, should be prepared in advance. (2) Compute the geometry information content of the terrain from the DEM files. (3) Compute the structure information content of the terrain from the DEM values. (4) DEM rendering: the basic rendered color 
ramp should be the same when at the same analysis. (5) Compute the pixel information content and the pixel difference information content of the terrain from a rendered DEM. (6) Compute the syntactic information content of the terrain from the geometry information, structure information, pixel information and pixel difference information contents. (7) Compute the terrain information parameters from DEM, i.e., SOA and SOS. (8) Compute the semantic information content from the terrain information parameters. (9) Compute the general information content of a terrain from the syntactic information content and the semantic information content. The whole process is shown in Figure 6, which presents the main flow chart describing how to extract the terrain information content from the DEM.

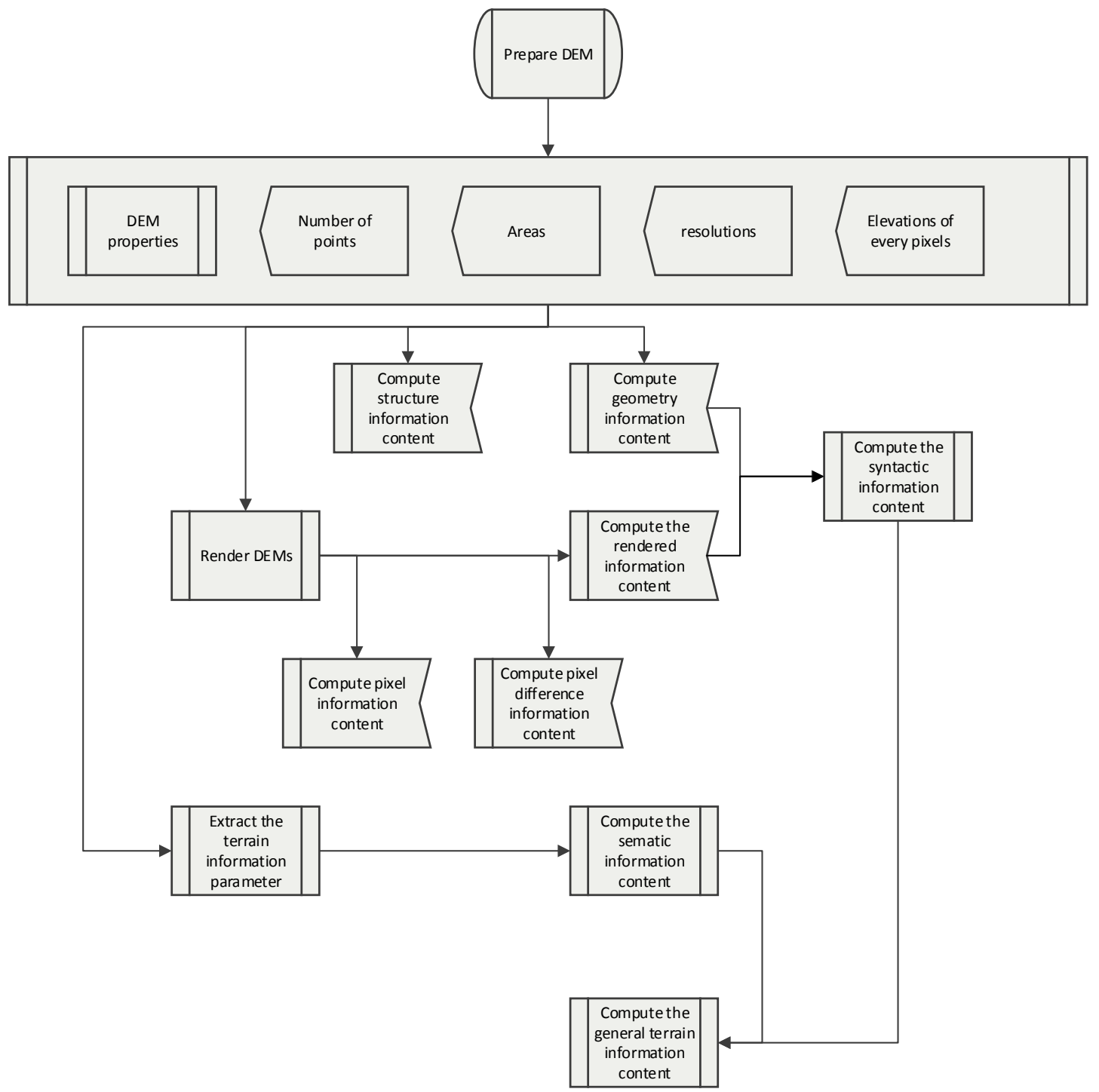

Figure 6. Flow chart for extracting terrain information content from a DEM.

\section{Experimental Analysis}

With simple logical deduction, we can presume two rules. One is that the more complex the terrain surface, the richer the terrain information. The other one is the finer the resolution, the richer the terrain information. The following will verify the two rules. 
Before verifying the two rules, we will introduce the DEM data quality first. The DEMs used are from the ASTER DEM. The resolution of the DEM is $30 \mathrm{~m}$. DEM data quality needed is different depending on different applications. The higher the data quality, the more accurate the terrain information content analysis. For the geomorphic analysis and resolution analysis, a unified data quality is necessary.

\subsection{Different Geomorphic Terrain Information Content Analysis}

There are seven basic geomorphic types of China [29], which are plain areas, tableland areas, hill areas, mountainous areas with low relief (low relief mountains), mountainous areas with moderate relief (moderate relief mountains), mountainous areas with high relief (high relief mountains), and mountainous areas with very high relief (very high relief mountains). For every geomorphic type, there is a relative elevation (relief height or elevation difference) range. If we use $H$ to represent the elevation difference (with units of meters), the data range of $H$ for different geomorphic types can be described as: for plain areas, $H<50 \mathrm{~m}$; for tableland areas, $H$ (especially the elevation difference between the table center and the table margin) $>500 \mathrm{~m}$; for hill areas, $H \in[50,200)$; for the low relief mountains, $H \in[200,500)$; for the moderate relief mountains, $H \in[500,1000)$; for the high relief mountains, $H \in[1000,2500)$; for the very high relief mountains, $H>2500 \mathrm{~m}$.

\subsubsection{Extraction of Results}

Before analyzing the relationship of terrain information content with different geomorphic types, we should determine the study area and the color ramp of rendering the DEM:

(1) Study area.

For every geomorphic type of China, specific areas are chosen as the study area, which are $40 \mathrm{~km} \times 40 \mathrm{~km}$. The following will describe the basic properties of each geomorphic area:

A. Plain area: For this type of geomorphic area, the area of the middle part of the Jiangsu plain in China is chosen as one of the study areas, for which the longitude is from $120.03^{\circ}$ to $120.39^{\circ}$, and the latitude is from $32.53^{\circ}$ to $32.835^{\circ}$.

B. Tableland area: For the tableland area, the middle part of Anhui Province in China is chosen. It is a low altitude and proluvial tableland. Its region is from longitude $117.05^{\circ}$ to $117.409^{\circ}$ and latitude $31.724^{\circ}$ to $32.03^{\circ}$.

C. Hills area: The experimental area chosen for this geomorphic type is located in the east of Sichuan Province and adjacent to Chongqing in China. It is a low altitude region, with longitude from $105.115^{\circ}$ to $105.474^{\circ}$ and latitude from $30.042^{\circ}$ to $30.354^{\circ}$.

D. Low Relief Mountains: The northeast of Liaoning Province is chosen as the study area for this geomorphic area. It has low elevation and lies within longitude $124.568^{\circ}$ to $124.927^{\circ}$ and latitude $42.026^{\circ}$ to $42.293^{\circ}$.

E. Moderate Relief Mountains: For this geomorphic experimental region, the area of north Guangxi Province and adjacent to the south of the province is selected. It is a middle elevation area, with longitude from $105.734^{\circ}$ to $106.093^{\circ}$ and latitude from $24.472^{\circ}$ to $24.802^{\circ}$. 
F. High Relief Mountains: This experimental area the area of northeast Qinghai Province adjacent to the southeast of Gansu Province is chosen. It is a high altitude area, with longitude from $101.908^{\circ}$ to $102.267^{\circ}$ and latitude from $37.178^{\circ}$ to $37.464^{\circ}$.

G. Very High Relief Mountains: For this types of geomorphic terrain, the chosen area is in the southeast of the Tibet plateau, lying in high altitude mountains. The longitude of this region is from $96.183^{\circ}$ to $96.543^{\circ}$, and the latitude is from $28.387^{\circ}$ to $28.703^{\circ}$.

Figure 7 shows the selected experimental areas of the different geomorphic types of terrain. The red rectangles, which are labeled from 1 to 7 , represent the experimental areas of the plains, tableland, hills, low relief mountains, moderate relief mountains, high relief mountains, and very high relief mountains in sequence.

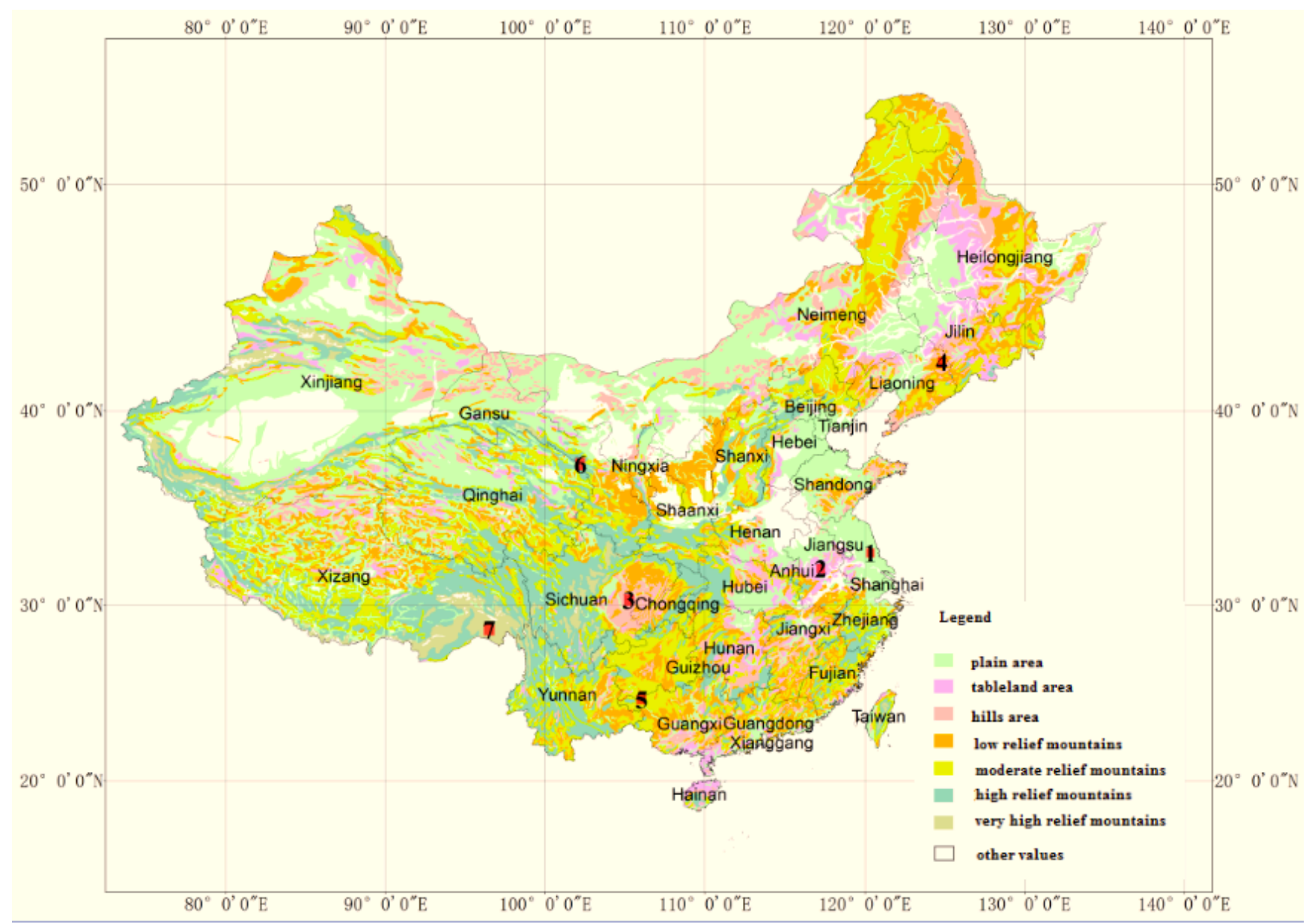

Figure 7. Distribution of the experimental areas for different geomorphic types of terrain.

\section{(2) DEM render color ramp}

Note that all of the DEMs should be rendered with a same color rendering method, which means that all of the seven areas should be mosaic to a whole DEM and be rendered with one rendering method. Alternatively, you can also extract the rendered DEM from a larger region which includes all of the seven DEMs, e.g., China's rendering terrain maps in the Web services. Figure 8 shows the rendering DEMs of the experimental areas and the color ramp. 


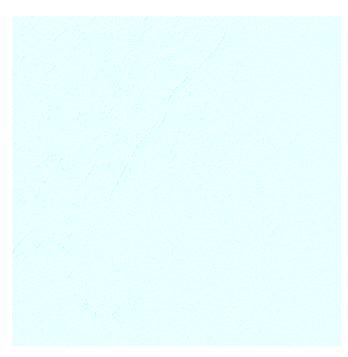

(a) Plain area

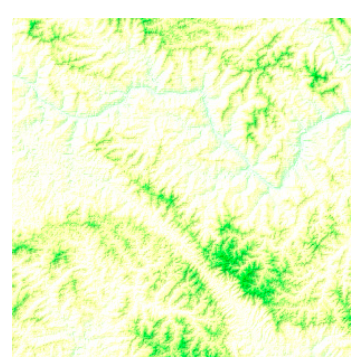

(e) Moderate relief mountains

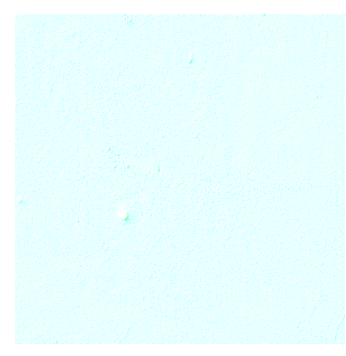

(b) Tableland area

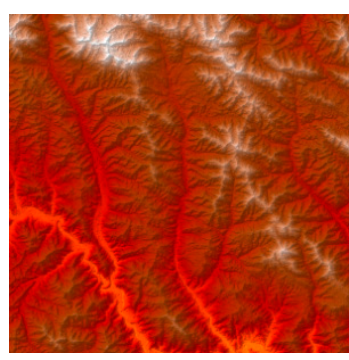

(f) High relief mountains

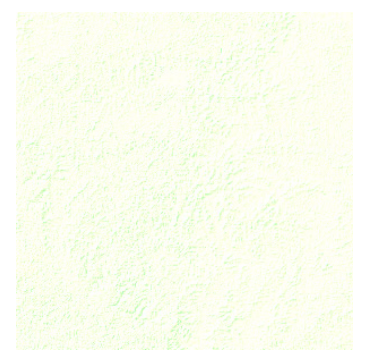

(c) Hills area

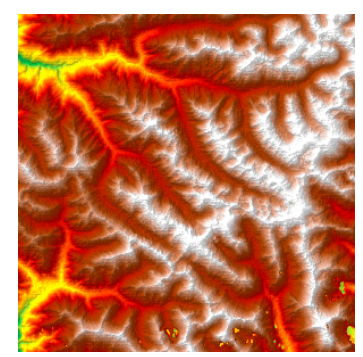

(g) Very high relief mountains

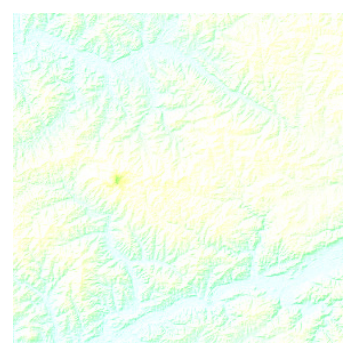

(d) Low relief mountains elevation ( $\mathrm{m}$ )

High : 5181

Low : -28

(h) Color ramp

Figure 8. Rendered digital elevation model (DEM) for different geomorphic types of terrains. All of the DEMs use the same color ramp, with the same minimum and high elevation values.

\section{(3) Results}

With the flow chart for the terrain information computation, we can extract the results of the terrain information content. The following Tables 1-3 show the syntactic information content, semantic information content and the general information content and their components' values for different geomorphic types.

Because the areas of all of the study regions are the same, as are the numbers of elevation points, the geometry information contents of these seven experimental areas are the same, as shown in Table 1. Moreover, note that the magnitude of the geometric information content value, the structure information content value and the rendered color information content value should be the same.

Table 1. Terrain syntactic information content of the seven different geomorphic types.

\begin{tabular}{ccccc}
\hline \multirow{2}{*}{ Geomorphic types } & \multicolumn{4}{c}{ Information content } \\
\cline { 2 - 5 } & $\boldsymbol{H}_{\boldsymbol{G E O} \boldsymbol{O}}$ & $\boldsymbol{H}_{\boldsymbol{S T R}}$ & $\boldsymbol{H}_{\boldsymbol{R E N}}$ & $\boldsymbol{H}_{\boldsymbol{S Y S}}$ \\
\hline plain area & 2.076 & 3.085 & 1.009 & 2.057 \\
tableland area & 2.076 & 6.014 & 1.244 & 3.111 \\
hills area & 2.076 & 7.538 & 1.670 & 3.761 \\
low relief mountains & 2.076 & 8.918 & 2.014 & 4.336 \\
moderate relief mountains & 2.076 & 9.386 & 2.498 & 4.653 \\
high relief mountains & 2.076 & 10.529 & 4.139 & 5.581 \\
very high relief mountains & 2.076 & 11.255 & 4.704 & 6.012 \\
\hline
\end{tabular}


Table 2. Terrain semantic information content and its subsets for the seven different geomorphic types.

\begin{tabular}{cccc}
\hline \multirow{2}{*}{ Geomorphic types } & \multicolumn{3}{c}{ Information content } \\
\cline { 2 - 4 } & $\boldsymbol{H}_{\boldsymbol{S O S}}$ & $\boldsymbol{H}_{\text {SOA }}$ & $\boldsymbol{H}_{\text {SEM }}$ \\
\hline plain area & 0.011446 & 2.657806 & 1.334626 \\
tableland area & 0.086478 & 2.725792 & 1.406135 \\
hills area & 0.784252 & 2.942306 & 1.863279 \\
low relief mountains & 0.86868 & 2.942426 & 1.905553 \\
moderate relief mountains & 1.357056 & 2.905492 & 2.131274 \\
high relief mountains & 1.407882 & 2.803122 & 2.105502 \\
very high relief mountains & 1.588496 & 2.618376 & 2.103436 \\
\hline
\end{tabular}

Table 3. General terrain information content of seven different geomorphic types.

\begin{tabular}{cc}
\hline Geomorphic types & Information content $\boldsymbol{H}_{\boldsymbol{T}}$ \\
\hline plain area & 3.391 \\
tableland area & 4.518 \\
hills area & 5.625 \\
low relief mountains & 6.242 \\
moderate relief mountains & 6.785 \\
high relief mountains & 7.687 \\
very high relief mountains & 8.115 \\
\hline
\end{tabular}

\subsubsection{Analysis of Results}

With computational results of different geomorphic terrain information contents, we can analyze the characteristics of the terrain information content from the follow aspects: (1) the syntactic information content with its components, and (2) the semantic information of the terrain with its components. Finally, the general information content is analyzed in total:

(1) Syntactic information content

From Table 1, the data trends can be plotted as follows. From Table 1 and Figure 9, the following points for the syntactic information content can be concluded: (I) Due to the experimental areas being all the same, the geometry information content is the same for every type of geomorphic terrain. (II) For information content other than the geometry information content, the more complex the geomorphic terrain type, the larger the values of the rendered, structure and the syntactic information contents. (III) The value of the structure information content is the maximum value among them.

Generally speaking, the variation trend of the structure, geometry, render and terrain syntactic information contents are in accord with the rules stated above. 


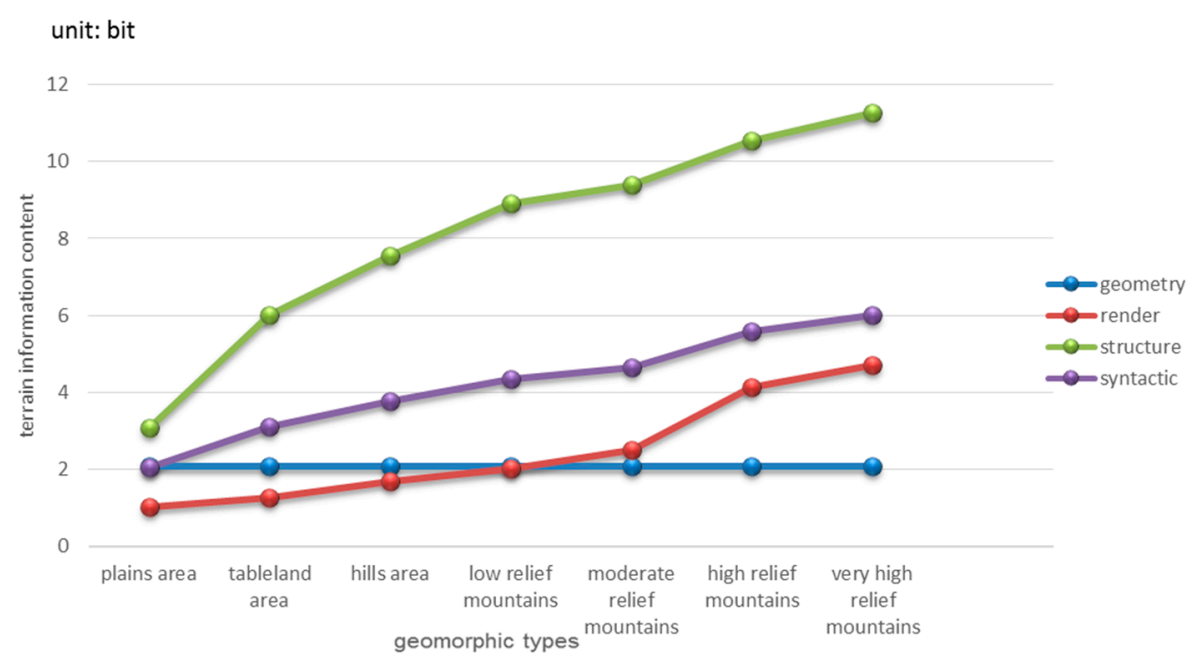

Figure 9. Data variation trends for the syntactic information content analysis for the seven geomorphic types.

\section{(2) Semantic information content}

From Table 2, the terrain semantic content data variation trends can be plotted as follows. From Table 2 and Figure 10, we can conclude the following: (I) The value of the information content of SOS increases with the terrain complexity from the plains area to the very high relief mountains. (II) The values of the SOA information content increase with the terrain complexity at first, and get the maximum value at the low relief mountains, then the values decrease with the terrain complexity. The low relief mountains area has the largest SOA information content value, and the hills area has a value of the SOA information content that is closer to the value for the low relief mountains area. They have two of the higher information content values of the geomorphic terrain types. (III) The semantic information content is combined with the characteristics of the SOA and SOS information contents. Except for the value of the medium relief mountain geomorphic type, the others increase progressively with the complexity of the geomorphic types. The information content for the medium relief mountain area is the largest value among them.

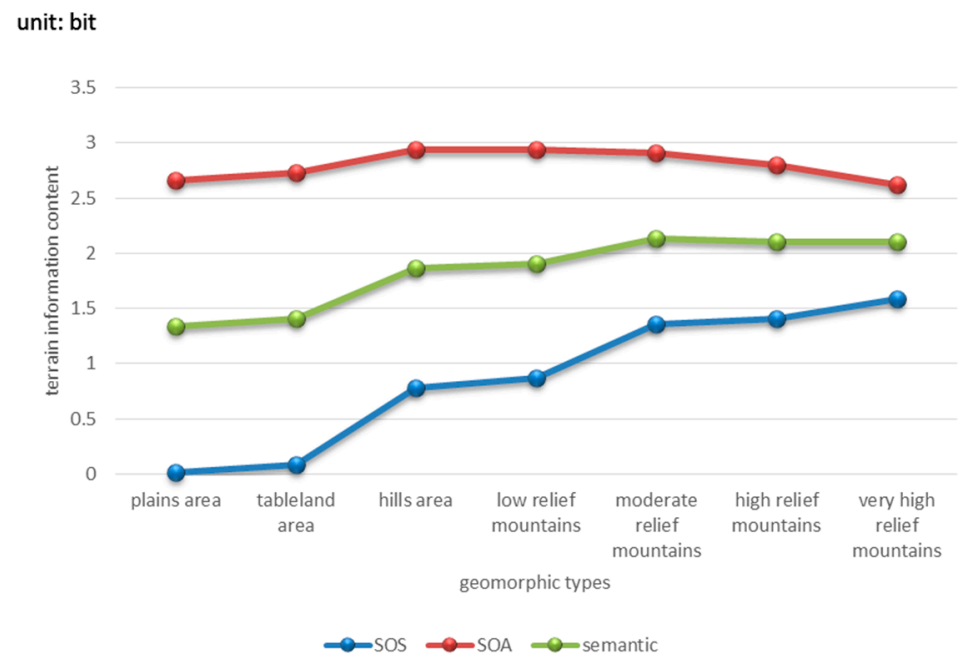

Figure 10. Data variation trend for the semantic information content analysis in the seven geomorphic types. 
Generally speaking, the information content of the semantic information content follows the rule where the more complex the terrain is, the larger the terrain information content. For the mountainous areas, the semantic information content values are larger than the values in the plain, tableland and hills areas. However, within the mountainous areas, the values are closely approximate.

(3) General information content

From Table 3, the variation trends of the general information content and its component information content can be plotted as in the following Figure 11. From Table 3 and Figure 11, we can conclude the following: (I) the value variation trends are mostly the same for the semantic information content, syntactic information content and the general information content. They all changed with the geomorphic terrain types: the more complex the geomorphic terrain type, the larger its information content value. (II) The semantic information content values are close to each other at the mountains areas (i.e., the small relief, medium relief, high relief and very high relief mountains).

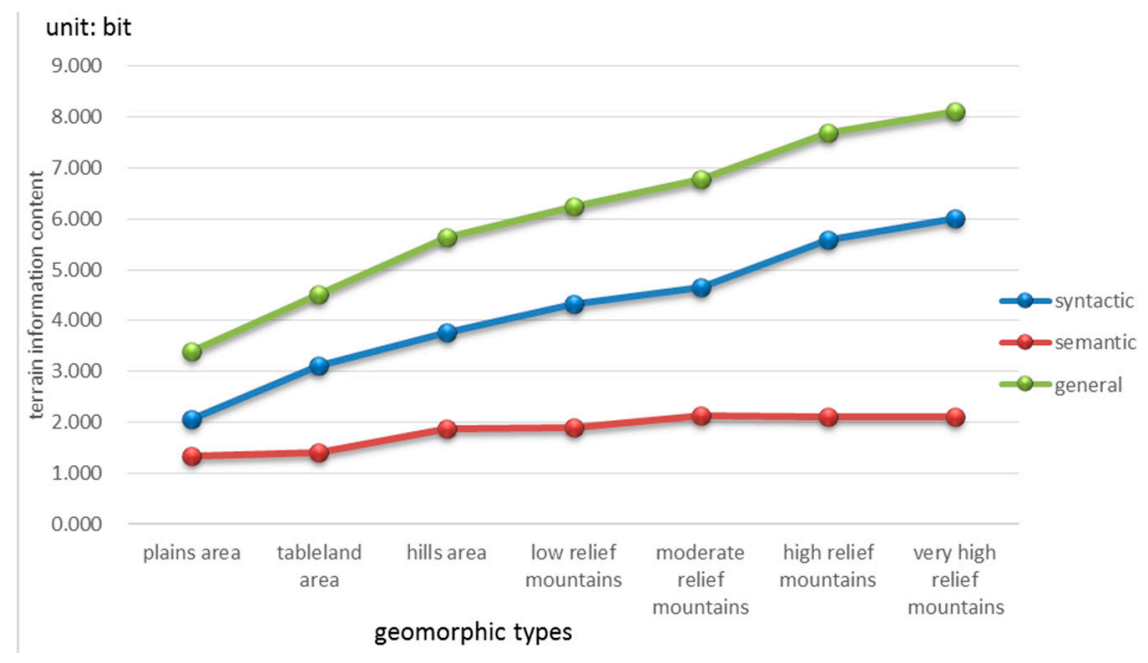

Figure 11. Data variation trend for the general terrain information content analysis for the seven geomorphic types.

Generally speaking, the terrain information content can reflect the terrain geomorphic types, and the terrain geomorphic types influenced the value of the terrain information content. This verifies the hypothesis that the more complex the terrain, the larger the information content value, and hence the richer the terrain information.

\subsection{Resolution Analysis}

\subsubsection{Extraction of Results}

With the flow chart for the computation of the terrain information content, the terrain information contents at different resolutions are computed. The resolutions we choose are $30 \mathrm{~m}, 60 \mathrm{~m}, 120 \mathrm{~m}$ and $240 \mathrm{~m}$.

Before analyzing the relationship between terrain information content and resolution, the following things should be determined: first, the study area we choose; and second, the color ramp for rendering the DEM, where all of the resolutions should be rendered with the same color ramp: 
(1) The hills area was chosen as the experimental study area for the resolution analysis because the hills area has rich terrain information, and it is a common terrain geomorphic type in China. The original experimental area has a resolution of $30 \mathrm{~m}$, and the resolutions of $60 \mathrm{~m}, 120 \mathrm{~m}$, and $240 \mathrm{~m}$ are resampled from the $30 \mathrm{~m}$ DEM with the bilinear interpolation method.

(2) The same color ramp used in the geomorphic analysis is chosen, as shown in Figure 12.

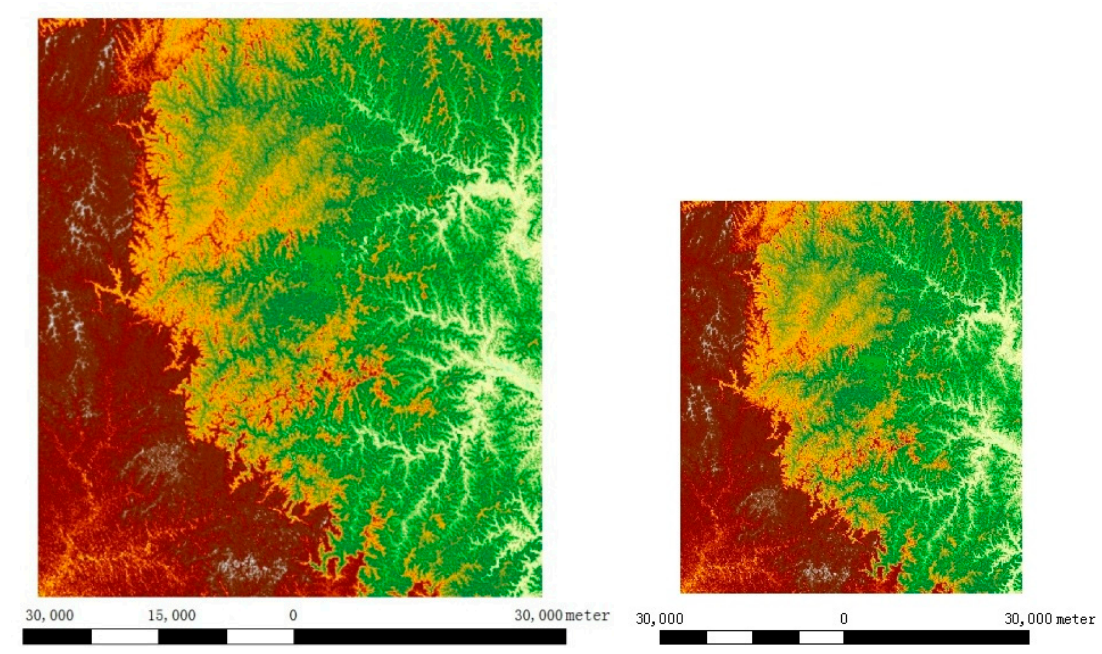

(a) $30 \mathrm{~m}$

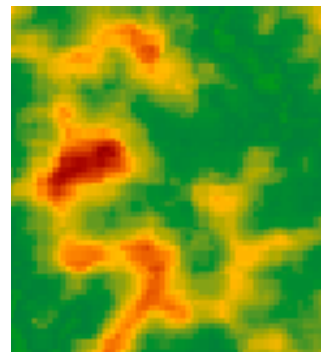

$\begin{array}{ll}\text { (e) Part region of } 30 \mathrm{~m} & \text { (f)Part region } 60 \mathrm{~m}\end{array}$ (b) $60 \mathrm{~m}$

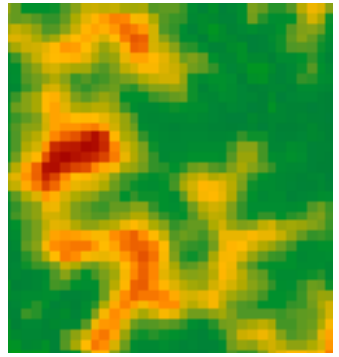

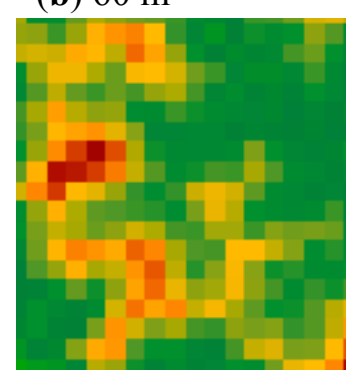

(g) Part region $120 \mathrm{~m}$

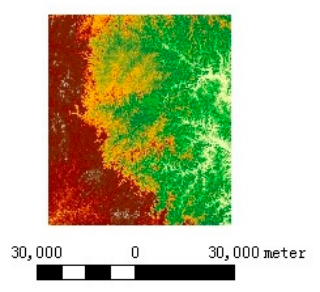

(c) $120 \mathrm{~m}$

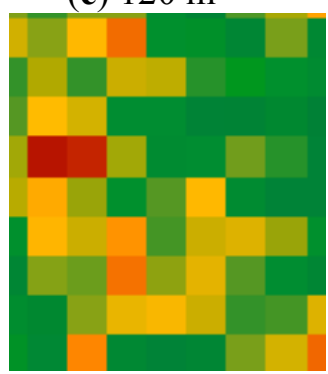

(h) Part region $240 \mathrm{~m}$

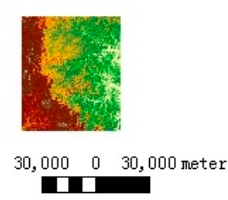

(d) $240 \mathrm{~m}$

elevation $(\mathrm{m})$

High : 586
Low : 223

(i) Color ramp

Figure 12. Rendered digital elevation model (DEM) of different resolutions. For each resolution, a small region in the right top of the origin DEM is enlarged to illustrate the differences of the resolutions.

With the four different resolution DEMs, terrain information content is extracted with the same method introduced above. The following tables show the syntactic information content, semantic information content and the general information content and their components' values. The magnitude of the geometric information content value is different from the other types of terrain information content values; we should minimize its magnitude and make it the same as the others. The geometric information content value should be reduced by a factor of 10; the following table shows the values that have been reduced by one order of magnitude. 
Table 4. Terrain syntactic information content and its components: geometry information content, structure information content, and render information content.

\begin{tabular}{ccccc}
\hline Resolution & $\boldsymbol{H}_{\boldsymbol{G E O} \boldsymbol{O}}$ & $\boldsymbol{H}_{\boldsymbol{S T R}}$ & $\boldsymbol{H}_{\boldsymbol{R E N}}$ & $\boldsymbol{H}_{\boldsymbol{S Y S}}$ \\
\hline $30 \mathrm{~m}$ & 2.192 & 7.790 & 4.246 & 4.743 \\
$60 \mathrm{~m}$ & 1.992 & 7.784 & 4.381 & 4.719 \\
$120 \mathrm{~m}$ & 1.792 & 7.783 & 4.450 & 4.675 \\
$240 \mathrm{~m}$ & 1.592 & 7.779 & 4.478 & 4.617 \\
\hline
\end{tabular}

Table 5. Terrain semantic information and its components: SOA information content and SOS information content.

\begin{tabular}{cccc}
\hline Resolution & $\boldsymbol{H}_{\text {SOA }}$ & $\boldsymbol{H}_{\text {SOS }}$ & $\boldsymbol{H}_{\text {SEM }}$ \\
\hline $30 \mathrm{~m}$ & 6.205 & 3.966 & 5.086 \\
$60 \mathrm{~m}$ & 5.957 & 2.544 & 4.251 \\
$120 \mathrm{~m}$ & 5.401 & 0.986 & 3.194 \\
$240 \mathrm{~m}$ & 4.597 & 0.021 & 2.309 \\
\hline
\end{tabular}

Table 6. General terrain information content.

\begin{tabular}{cc}
\hline Resolution & $\boldsymbol{H}_{\boldsymbol{T}}$ \\
\hline $30 \mathrm{~m}$ & 9.828 \\
$60 \mathrm{~m}$ & 8.969 \\
$120 \mathrm{~m}$ & 7.869 \\
$240 \mathrm{~m}$ & 6.925 \\
\hline
\end{tabular}

\subsubsection{Result Analysis}

With the results of the terrain information content at different resolutions, we can analyze from the following respects: first, the influence of resolution on the syntactic information content and its components (i.e., geometric information content, structure information content and rendered information content); second, the influence of resolution on the semantic information and its components (SOA information content and the SOS information content); and the influence of resolution on the general information content:

(1) Syntactic information content

From Table 4, the value variation trend of the syntactic information content with its components can be generated as in Figure 13. 


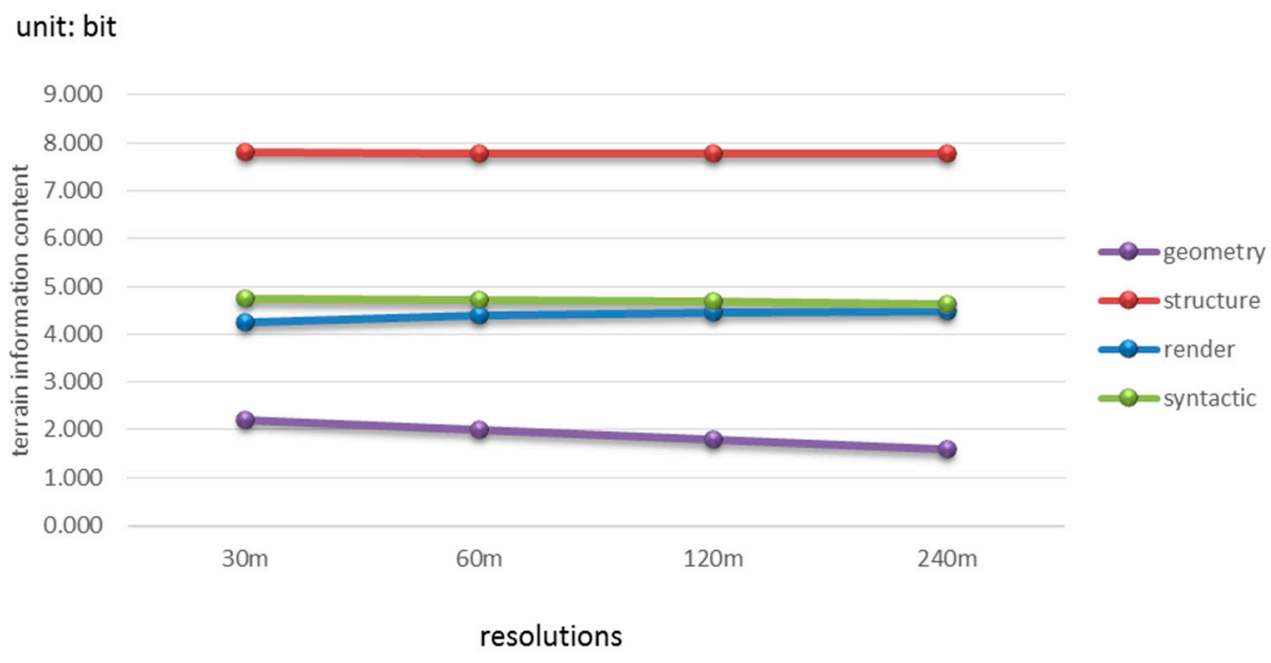

Figure 13. Data variation trend for the terrain syntactic information content analysis with different resolutions.

From the Figure 13 and the data in Table 4 for the syntactic information content, the following can be concluded: (I) Except for the render information content, the values of other information contents are in accordance with the variation roles, the finer the resolution, the larger the value of the information content. The resolution of $30 \mathrm{~m}$ has the richest terrain information. (II) The render information content rises as the resolution is decreased. The reason for that may be because the lower the precision of a picture, the higher the uncertainty.

(2) Semantic information content

A variation trend can be drawn from Table 5, as shown in Figure 14, for the semantic information content and its components, which are SOA information content and the SOS information content.

unit: bit

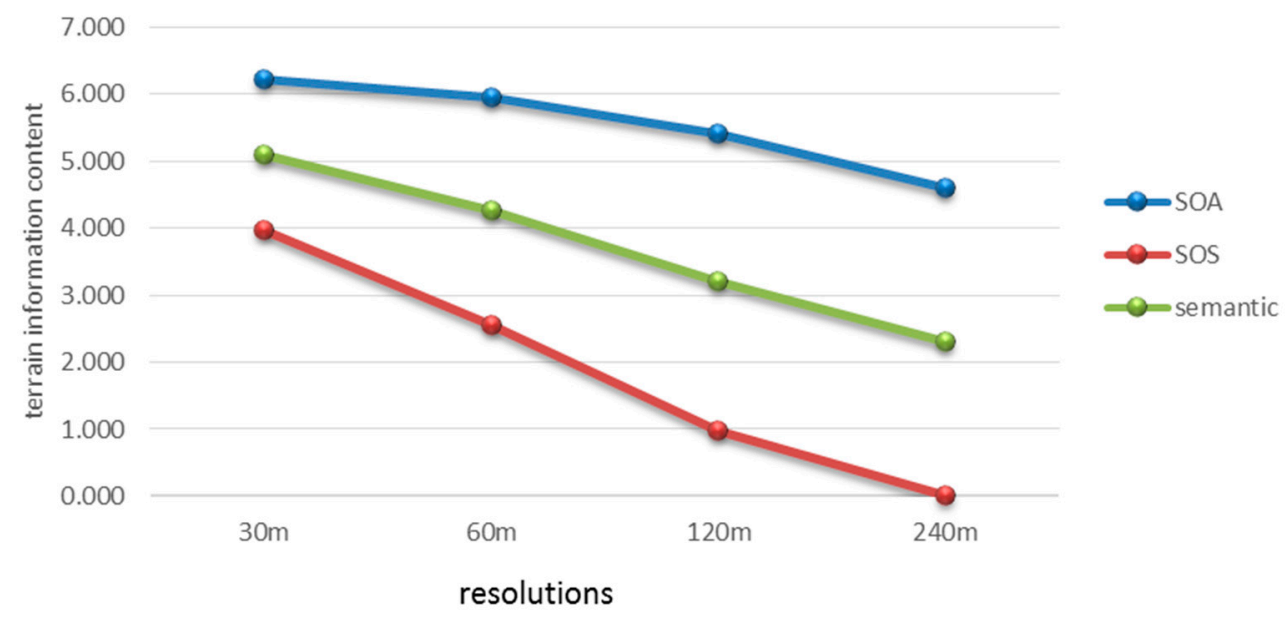

Figure 14. Data variation trend for the terrain semantic information content analysis with different resolutions.

From the above Figure 14 and the data of Table 5, we can conclude the following for the semantic information content: (I) The SOA information content, SOS information content and the semantic 
information content values all follow the rules where the larger the information content value, the finer the resolution. (II) The information content of the SOA is larger than that of the SOS, which means that the terrain information is richer from the horizontal variation.

(3) General information content

From Tables 4-6, the value variation trend for the terrain syntactic information content, semantic information content, and the general information content can be generated, as shown in Figure 15.

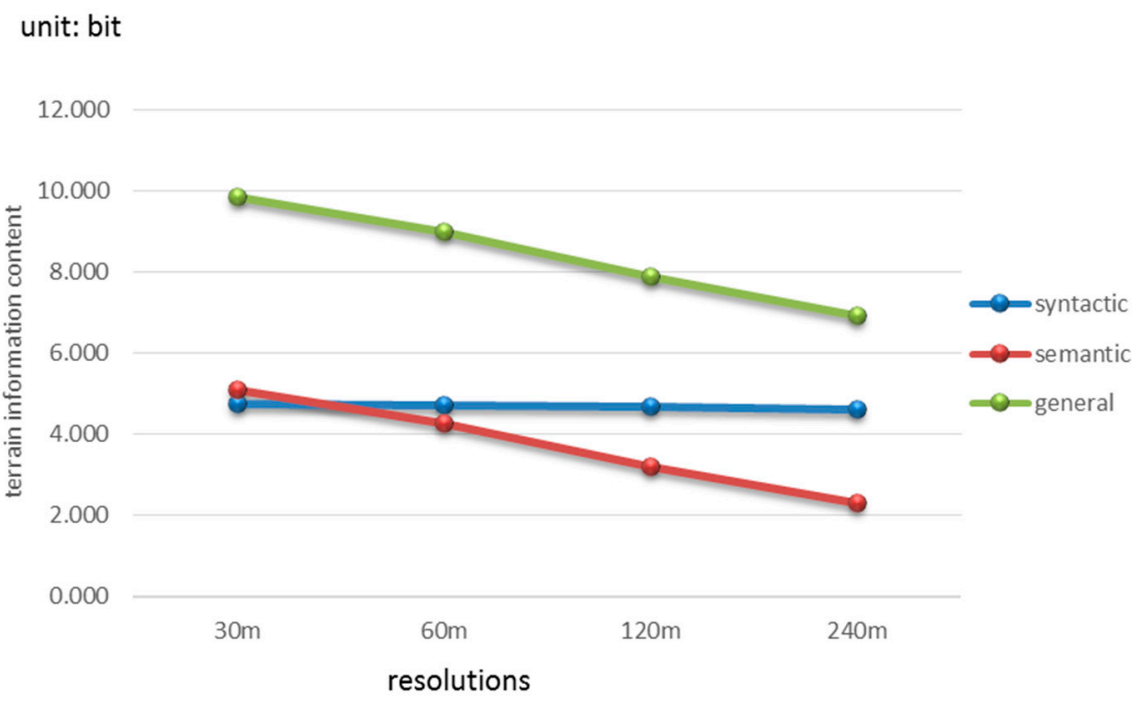

Figure 15. Data variation trend for the general terrain information content analysis with different resolutions.

As Figure 15 and Tables 4-6 show, the general information content for the terrain shows the following characteristics: (I) With the decrease of the resolution, the value of the syntactic information content, the semantic information content and the general information content are also decreased. (II) The decrease rate of the information content value is larger for the semantic information content than for the syntactic information content.

Generally speaking, the terrain information content is clearly influenced by the DEM resolutions, i.e., the finer the resolution, the richer the terrain information content. The opposite is true for the same experimental area: when the information content value is larger, we can judge that the resolution of the DEM is larger.

\section{Discussion and Conclusions}

Based on the theories of the map information content, remote sensing image information content and other geospatial information content, a definition and measures for extracting the terrain information content from DEM data are proposed in this paper. Moreover, experiments are carried out from two aspects: one is the analysis of terrain information content in different geomorphic types, and the other is the analysis of terrain information content with different resolutions. The geomorphic types analysis showed that the more complex the terrain surface, the richer the information content. The resolution analysis showed that the finer the resolution, the richer the terrain information content. Both of the experiments reflected the reliability of the measurement method for the terrain information content. 
The definition and measurement method for the terrain information content is different from the traditional measurement method for geospatial information content. The existing measurement method for geospatial information content is always computed from the feature maps (e.g., transport, topographic, and contour maps). However, the terrain information content proposed in this paper is one type of geospatial information content; it was computed from a rendered DEM and is a supplement to the geospatial information content. Moreover, the terrain information content is also superior to the information measurement method for DEM in some specific applications. The existing information measures with DEM always focus on elevation entropy and terrain factor's entropy. Elevation entropy is related to the subset of the elevation dataset and its elevation dataset partition way (i.e., terrain structure information content defined in our paper). Terrain factor's entropy is the value entropy of the terrain factor (e.g., roughness, curviness of terrain) extracted from DEM. It is related to the subset of the terrain factor value and its partition method. Elevation entropy and terrain factor's entropy computed from DEM usually applied to the following applications. For example, in the research of Wise [30], the elevation entropy is used for DEM quality evaluation, particular the DEM information loss when aggregating the DEM to a coarser scale. In reference [31], the elevation entropy is applied to determine the optimum resolution of DEM in prior to hydrological modeling. In reference [32], entropy of terrain curvature, as a measure of information loss at different scales, are useful for correcting conductivity values when upscaling the subsurface flow equation in macro scale hydrological modeling. In reference [33], elevation corrections entropy is used to evaluate the information loss of the DEM interpolation method. However, terrain information content will improve the way of information evolution applied to DEM described above. Its superiority can be described as follows.

Based on the theory of the epistemology, two levels of terrain information content are introduced, which are terrain syntactic information content and terrain semantic information content. The general terrain information content is the combination of these two. For the two levels of terrain information content, they are also constituted by some other information, as shown in Figure 16. In the first level of terrain information content, the rendering information content can be accessed by a combination with other visual method for some specific applications, e.g., bipolar differentiation visual method [29] for geomorphic analysis, or analytical shading method for 3-dimensional analysis. Generally, we use the general information content for the most of the applications, like optimizing the terrain generalization method. However, according to your data source and your demands, you can use them individually or with an arbitrary combination. For example, when evaluate the DEM quality in reference [30], the elevation entropy can reflect the elevation variation, but it cannot represent the potential meaning of this variation. Hence, if the measurement method is combined terrain semantic information with the terrain structure information, it will better reflect the DEM quality and the information loss when aggregate it. For the application of finding the optimize resolution in the hydrological modelling [31], the best resolution will be determined by the least terrain information loss when DEM was aggregated from fine scale, so the entropy of terrain curvature can be replaced by the terrain semantic information content, which has a better represent of the terrain variation in horizontal and vertical aspect. 


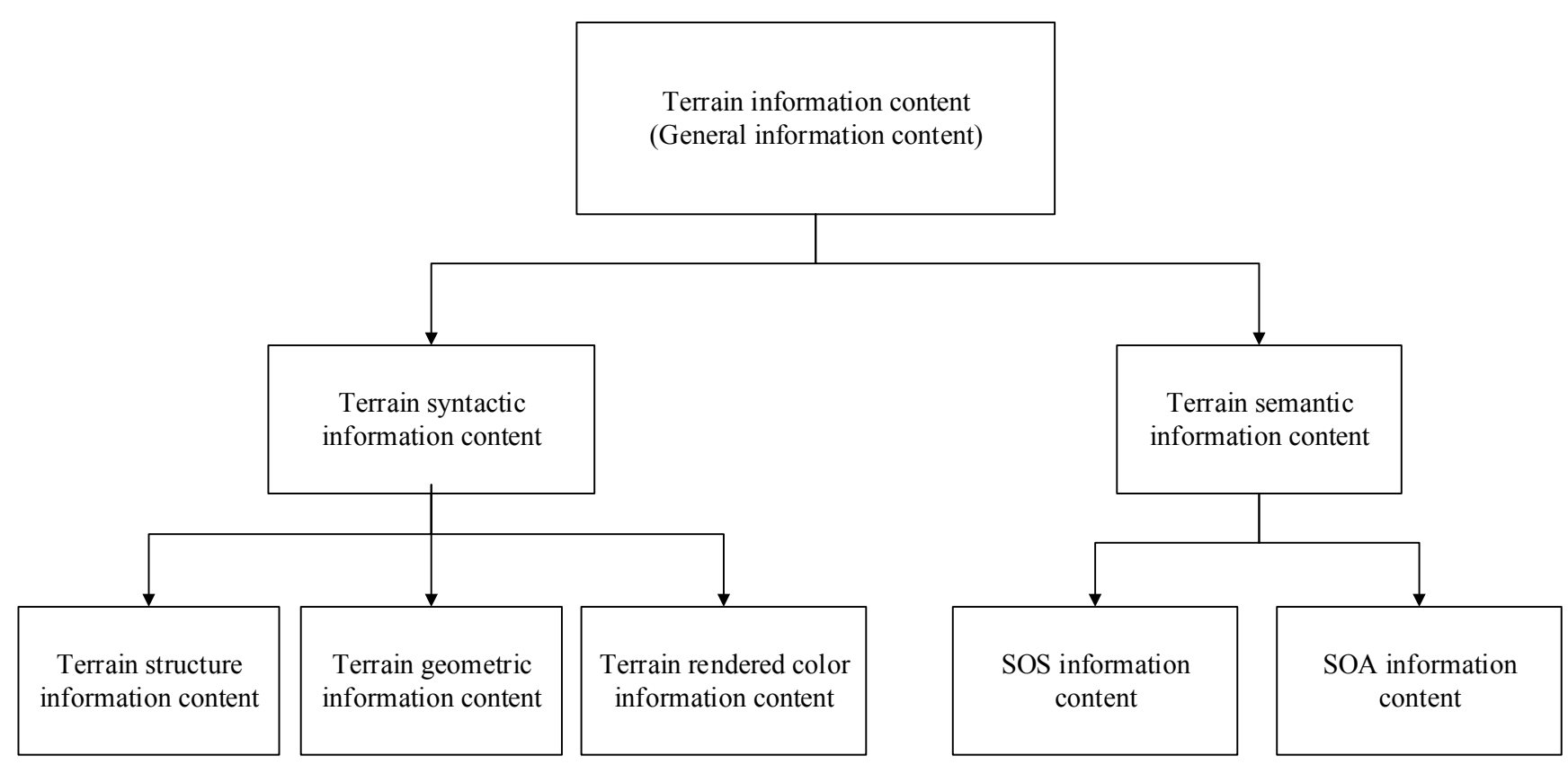

Figure 16. Framework and components for the terrain information content.

Besides these applications above, there are many other applications that could use the terrain information content, and all of these applications are needed intensive studies in the future. For example, the rendered color information content can be used for evaluating the quality of the rendered DEM tile service from some famous websites. The larger the rendered color information content, the better the terrain map rendering quality. As shown in Figure 17, two kinds of rendered terrain maps are extracted from the websites of Google Maps and Map World. The two rendered terrain maps are using completely different color rendering methods. From the rendering surface of the two maps, we may feel the Google Maps renders better. The results we calculated from our terrain information tool showed that the values of the rendered color information content are 7.19 bits and 7.59 bits for Figure 17a and b, respectively. Hence we can conclude that the rendered color information content may represent the rendered terrain map quality. For another example, the terrain information content can also be used as a new way of dividing the basic geomorphic types, which is traditionally divided by the relative elevation. Statistics of the general terrain information content can be extracted from different geomorphic types. Then we can analyze and define the value threshold of terrain information content for different geomorphic types. Meanwhile it is necessary to study the relation between terrain information content and the different geomorphic types classified by Landform Genesis, e.g., Danxia landform, Karst landform, Yardang landform (wind-erosion landform). Furthermore, other visual method (as stated in reference [34]) would be taken into account as one way of the rendering method for DEM to access the rendering color information content. We should study whether we can combine them into some specific analysis to improve the effectiveness of the analysis result. e.g., with the bipolar differentiation visual method to render the DEM for the geomorphic analysis, as it is sensitive to different geomorphic types. In short, future works will be focused on the specific applications for the terrain information content above. 


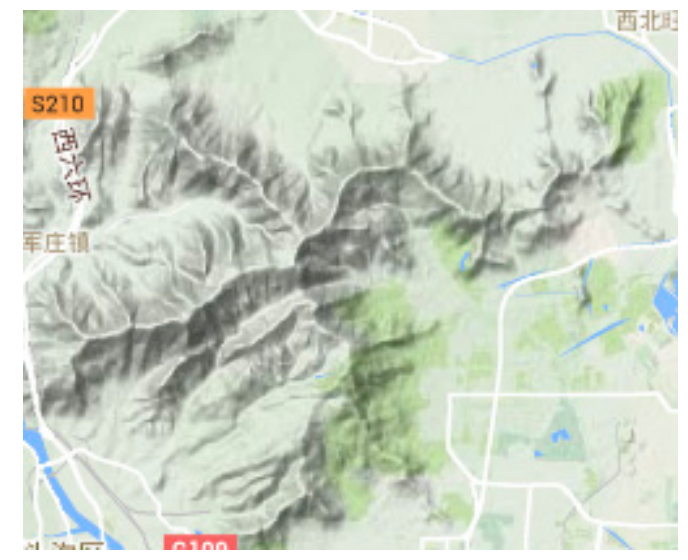

(a) Terrain rendered tile maps of Google Maps

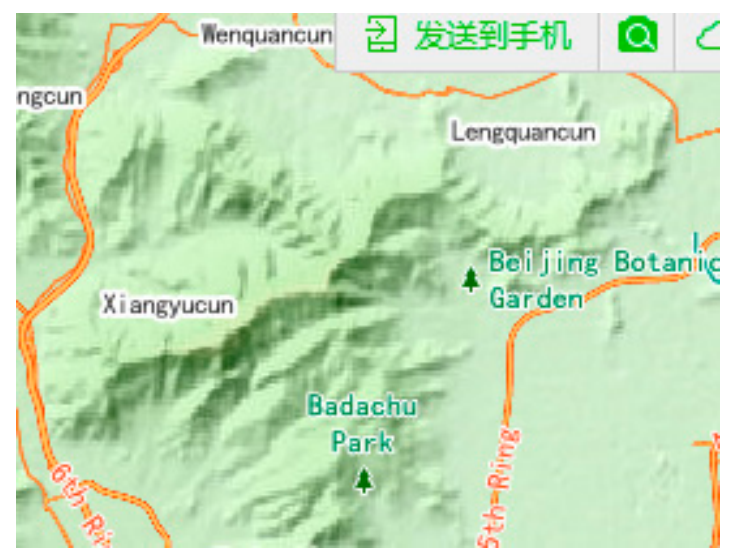

(b) Terrain rendered tile maps of Map World

Figure 17. Rendered terrain tile maps from Google maps and Map word of China. They are rendered with completely different color ramp.

\section{Acknowledgments}

This work was supported by the Fundamental Research Funds for the Central Universities, No. 2014205020203, and National 863 Project of China, No. G1213. Agen Qiu and Kunwang Tao have provided plenty of suggestions for the article, as well as providing careful review; we express our grateful appreciation here for their significant contributions to this article.

\section{Author Contributions}

Lujin $\mathrm{Hu}$ conceived and designed the methodology of terrain information content, and realized the terrain information computation tools. Zongyi He and Jiping Liu advised for the method of terrain information content, and did the part of the experimental analysis. Chunhua Zheng provided the basic information entropy calculation method in the information theory for this paper, and advised for the method of terrain information content. All authors have read and approved the final manuscript.

\section{Conflicts of Interest}

The authors declare no conflict of interest.

\section{References}

1. Li, Z.; Zhu, Q.; Gold, C. Digital Terrain Modeling: Principles and Methodology; CRC Press: Boca Raton, FL, USA, 2005.

2. Hutchinson, M.F.; Gallant, J.C. Digital elevation models and representation of terrain shape. In Terrain Analysis, Principles and Applications; Wiley: New York, NY, USA, 2000.

3. Hengl, T.; Reuter, H.I. Geomorphometry: Concepts, Software, Applications; Elsevier: Amsterdam, The Netherlands, 2009.

4. Wilson, J. P.; Gallant, J.C. Terrain Analysis: Principles and Applications; Wiley: New York, NY, USA, 2000. 
5. Zhou, Q.; Lees, B.; Tang, G. Advances in Digital Terrain Analysis; Springer: Berlin/Heidelberg, Germany, 2008.

6. Lazaro, J.M.; Navarro, J.A.S.; Gil, A.G.; Romero, V.E. Developing and programming a watershed traversal algorithm (WTA) in GRID-DEM and adapting it to hydrological processes. Comput. Geosci. 2013, 51, 418-429.

7. Gao, M.; Zeilinger, G.; Xu, X.; Wang, Q.; Hao, M. DEM and GIS analysis of geomorphic indices for evaluating recent uplift of the northeastern margin of the Tibetan Plateau, China. Geomorphology 2013, 190, 61-72.

8. Clark, D. Identification of Quaternary scarps in southwest and central west Western Australia using DEM-based hill shading: Application to seismic hazard assessment and neotectonics. Int. J. Remote Sens. 2010, 31, 6297-6325.

9. Sukhov, V.I. Information capacity of a map entropy. Geod. Aerophotogr. 1967, 10, 212-215.

10. Sukhov, V.I. Application of information theory in generalization of map contents. Int. Yearb. Cartogr. 1970, 10, 41-47.

11. Fairbairn, D. Measuring map complexity. Cartogr. J. 2006, 43, 224-238.

12. Llibre, J.; Saghin, R. Results and open questions on some invariants measuring the dynamical complexity of a map. Fundam. Math. 2009, 206, 307-327.

13. Harrie, L.; Stigmar, H. An evaluation of measures for quantifying map information. ISPRS $J$. Photogramm. Remote Sens. 2010, 65, 266-274.

14. Bjørke, J.T. Framework for entropy-based map evaluation. Cartogr. Geogr. Inf. Syst. 1996, 23, $78-95$.

15. Li, Z.L.; Huang, P.Z. Quantitative measures for spatial information of maps. Int. J. Geogr. Inf. Sci. 2002, 16, 699-709.

16. Neumann, J. Gnoseological aspects of improving the geometrical component of the space-time model in cartography. In Proceedings of the 13th International Cartographic Conference ICA, Morelia, Mexico, 12-21 October 1987; pp. 569-584.

17. Neumann, J. The topological information content of a map/an attempt at a rehabilitation of information theory in cartography. Cartographica 1994, 31, 26-34.

18. Lin, Z.; Zhang, Y. Measurement of information and uncertainty of remote sensing and GIS data. Geomat. Inf. Sci. Wuhan Univ. 2006, 31, 569-572.

19. Lin, Z.; Deng, B. Quantifying Degrees of Information in Remote Sensing Imagery. In Proceedings of the 8th International Symposium on Spatial Accuracy Assessment in Natural Resources and Environmental Sciences, Shanghai, China, 25-27 June 2008; pp. 201-205.

20. Chen, Y.; Sun, K.; Lin, Z. Measures of information in remote sensing imagery. J. Comput. Inf. Syst. 2011, 7, 1648-1654.

21. Ruiz, M.; Lopez, F.; Paez, A. Comparison of thematic maps using symbolic entropy. Int. J. Geogr. Inf. Sci. 2012, 26, 413-439.

22. Novoa, J.; Chokmani, K.; Nigel, R.; Dufour, P. Quality assessment from a hydrological perspective of a digital elevation model derived from WorldView-2 remote sensing data. Hydrol. Sci. J. 2015, 60, 218-233.

23. Chen, X.; Hu, T.; Ren, F.; Chen, D.; Li, L.; Gao, N. Landscape analysis of geographical names in Hubei province, China. Entropy 2014, 16, 6313-6337. 
24. Shannon, C.E.; Weaver, W. The Mathematical Theory of Communication; The University of Illinois Press: Urbana, IL, USA, 1964.

25. Head, C.G. Mapping as language or semiotic system: Review and comment. In Cognitive and Linguistic Aspects of Geographic Space; Mark, D.M., Frank, A.U., Eds.; Springer: Amsterdam, The Netherlands, 1991; Volume 63, pp. 237-262.

26. Tang, G.; Yang, X. ArcGIS Geographic Information System Spatial Analysis Experiment Course; Science Press: Beijing, China, 2006.

27. ArcGIS Help Library 10.1. Available online: http://resources.arcgis.com/en/help/main/10.1/ index.html\#//00qn0000001p000000 (accessed on 10 September 2015).

28. Burrough, P.A.; McDonell, R.A. Principles of Geographical Information Systems; Oxford University Press: New York, NY, USA, 1998; p. 190.

29. Li, B.; Pan, B.; Cheng, W.; Han, J.; Qi, D.; Zhu, C. Research on geomorphological regionalization of China. Acta Geogr. Sin. 2013, 68, 291-306.

30. Wise, S. Information entropy as a measure of DEM quality. Comput. Geosci. 2012, 48, 102-110.

31. Sharma, A.; Tiwari, K.N.; Bhadoria, P.B.S. Determining the optimum cell size of digital elevation model for hydrologic application. J. Earth Syst. Sci. 2011, 120, 573-582.

32. Niedda, M. Upscaling hydraulic conductivity by means of entropy of terrain curvature representation. Water Resour. Res. 2004, 40, doi:10.1029/2003WR002721.

33. Kidner, D.; Dorey, M.; Smith, D. What's the point? Interpolation and extrapolation with a regular grid DEM. In Proceedings of the 4th International Conference on GeoComputation, Fredericksburg, VA, USA, 25-28 July 1999.

34. Podobnikar, T. Methods for visual quality assessment of a digital terrain mode. SAPI EN. S. Surveys Perspect. Integr. Environ. Soc. 2009, 2, 15-24.

(C) 2015 by the authors; licensee MDPI, Basel, Switzerland. This article is an open access article distributed under the terms and conditions of the Creative Commons Attribution license (http://creativecommons.org/licenses/by/4.0/). 